\title{
REDUCTION OF DIRAC STRUCTURES AND THE HAMILTON-PONTRYAGIN PRINCIPLE
}

\author{
HIROAKI YOSHIMURA* \\ Department of Mechanical Engineering, Waseda University, Ohkubo, Shinjuku, Tokyo 169-8555, Japan \\ (e-mail: yoshimura@waseda.jp) \\ and \\ JERROLD E. MARSDEN $\dagger$ \\ Control and Dynamical Systems, California Institute of Technology, Pasadena, CA 91125, USA \\ (e-mail: marsden@cds.caltech.edu)
}

(Received February 19, 2007 - Revised September 17, 2007)

This paper develops a reduction theory for Dirac structures that includes in a unified way, reduction of both Lagrangian and Hamiltonian systems. It includes the reduction of variational principles and in particular, the Hamilton-Pontryagin variational principle. It also includes reduction theory for implicit Lagrangian systems that could be degenerate and have constraints.

In this paper we focus on the special case in which the configuration manifold is a Lie group $G$. In our earlier papers we established the link between the Hamilton-Pontryagin principle and Dirac structures. We begin the paper with the reduction of this principle. The traditional view of Poisson reduction in this case is to reduce $T^{*} G$ with its natural Poisson structure to $\mathfrak{g}^{*}$ with its Lie-Poisson structure. However, the basic step of reducing Hamilton's phase space principle already shows that it is important to use $\mathfrak{g} \oplus \mathfrak{g}^{*}$ for the reduced space, rather than just $\mathfrak{g}^{*}$. In this way, our construction includes both Euler-Poincaré as well as Lie-Poisson reduction. The geometry behind this procedure, which we call Lie-Dirac reduction starts with the standard (i.e., canonical) Dirac structure on $T^{*} G$ (which can be viewed either symplectically or from the Poisson viewpoint) and for each $\mu \in \mathfrak{g}^{*}$, produces a Dirac structure on $\mathfrak{g} \oplus \mathfrak{g}^{*}$. This geometry then simultaneously supports both Euler-Poincaré and Lie-Poisson reduction.

In the last part of the paper, we include nonholonomic constraints, and illustrate this construction with Suslov systems in nonholonomic mechanics, both from the Euler-Poincaré and Lie-Poisson viewpoints.

Keywords: reduced Hamilton-Pontryagin principle, Lie-Dirac reduction, implicit Lagrangian systems, Suslov problems.

\section{Introduction}

Dirac structures aim to synthesize Poisson structures and pre-symplectic structures. The idea was originally developed by Courant and Weinstein [25], taking some

\footnotetext{
*Research partially supported by JSPS Grant 16560216.

$\dagger$ Research partially supported by NSF-ITR Grant ACI-0204932.
} 
inspiration from Dirac's theory of constraints for degenerate Lagrangians together with associated Poisson brackets (see also Courant [23]). In mechanics, Dirac structures fit fairly readily into the framework of Hamilton mechanics and, correspondingly, the notion of an implicit Hamiltonian system associated with a Dirac structure on a manifold was developed by van der Schaft and Maschke [47], where energyconserving systems such as L-C circuits and nonholonomic systems were put into the context of Dirac structures (see also Bloch and Crouch [8]).

Recently, the notion of an implicit Lagrangian system, which is a Lagrangian analogue of an implicit Hamiltonian system, was developed by Yoshimura and Marsden [50], where it was shown that nonholonomic mechanical systems and L-C circuits as degenerate Lagrangian systems can be formulated in this context.

Furthermore, the link between variational principles and Dirac structures was established and for the case in which a given Lagrangian is regular, an implicit Hamiltonian system can be derived, as in Yoshimura and Marsden [51]. This was done, via a generalized Legendre transformation, for the case of degenerate Lagrangian systems as well, in Yoshimura and Marsden [52].

Throughout this paper we shall draw on some developments in the papers of Yoshimura and Marsden [50, 51]. These include the notion of implicit Euler-Lagrange equations and how these equations are related to a Dirac structure and a given Lagrangian. We will also need to know how these equations are related to an extended variational principle of Hamilton called the Hamilton-Pontryagin principle. In addition, we will need to draw on the notion of a Lagrangian system associated with an induced Dirac structure in case one has a nontrivial constraint distribution (perhaps nonholonomic) and how it is related to the Lagrange-d'Alembert-Pontryagin variational principle.

One approach to the reduction of Dirac structure was given by Courant [23], consistent with Poisson reduction developed by Marsden and Ratiu [39] and also by Dorfman [28] in conjunction with infinite dimensional Hamiltonian systems. Along similar lines, the symmetry reduction of Dirac structures and its associated implicit Hamiltonian systems was developed by van der Schaft [48]; Blankenstein and van der Schaft [5]. Those previous reduction procedures were basically related with the symplectic reduction developed by Marsden and Weinstein [42] or the corresponding Poisson reduction procedures. Later in this introduction, we shall briefly return to this topic and give additional history and literature.

\section{Goal}

The overall purpose of this work is to establish a geometric reduction theory for Dirac structures that supports the reduction of implicit Lagrangian systems as well as reduction of implicit Hamiltonian systems and the variational structures associated to each. As we shall see, the reduction method is necessarily somewhat different than those treated previously. The present paper is focused on the case in which the configuration manifold is a Lie group $G$. Future papers will deal with more general configuration manifolds with group actions. 
Specifically, the paper focuses on the reduction of the canonical Dirac structure on the cotangent bundle $T^{*} G$ of a Lie group $G$. Our construction supports both the Lagrangian and Hamiltonian viewpoints, giving Euler-Poincaré-Dirac reduction when one takes the Lagrangian view, and Lie-Poisson-Dirac reduction when one takes the Hamiltonian view. As we mentioned, our treatment of implicit Lagrangian systems via Dirac structures is consistent with the Hamilton-Pontryagin variational principle. Consistent with the reduction of the Dirac structure itself, of implicit Lagrangian and Hamiltonian systems, we also reduce the Hamilton-Pontryagin principle. Towards the end of the paper, systems with nonholonomic constraints are studied and an example of nonholonomic rigid body mechanics is examined, which leads to a reduction method associated with Suslov systems.

\section{Key differences with other Dirac reduction schemes}

Below we review some of the main literature on reduction of Dirac structures. The key difference between the present work and those is, as we have mentioned, the fact that our approach accommodates a Lagrangian, a Hamiltonian, and a variational view simultaneously. To do so, one needs to work with slightly larger spaces, as is already evident in the reduction of Hamilton's phase space principle, to give a variational principle for the Lie-Poisson equations, which was developed in Cendra, Marsden, Pekarsky and Ratiu [18]. Specifically, many schemes in the literature take the following view (or a variant of it): one starts with a manifold $M$, an (almost) Dirac structure on $M$, and a group $G$ acting on $M$ (consistent with the Dirac structure). From these ingredients, one then constructs a Dirac structure on $M / G$. For example, for $M=T^{*} G$ with $G$ acting by group multiplication, one gets $\mathfrak{g}^{*}$ with the Dirac structure associated with the Lie-Poisson structure. However, this construction is too limited for our purposes and does not allow one to include variational principles or the Lagrangian view. In our case of mechanics on Lie groups, the basic difference in our approach is that instead of $\mathfrak{g}^{*}$, the resulting reduced space is $\mathfrak{g} \oplus \mathfrak{g}^{*}$.

\section{Background on reduction}

Reduction theory has a rich history in mechanics and there are a lot of research developments, which may be found in, for instance, Marsden and Ratiu [40], Marsden, Ratiu and Scheurle [41], Cendra, Marsden, and Ratiu [20], Marsden and Weinstein [44], Marsden, Misiolek et. al. [38]. We next briefly review symplectic and Poisson reduction with emphasis on the special case of the cotangent bundle of a Lie group, which is the case most relevant for the present paper.

Let $(P, \Omega)$ be a symplectic manifold and let $G$ be a Lie group that acts freely and properly on $P$ by symplectic maps. Suppose that the action has an equivariant momentum map $\mathbf{J}: P \rightarrow \mathfrak{g}^{*}$ and let $G_{\mu}:=\left\{g \in G \mid \operatorname{Ad}_{g^{-1}}^{*} \mu=\mu\right\}$ be the coadjoint isotropy subgroup of $\mu \in \mathfrak{g}^{*}$. Then, one can obtain the symplectic reduced space 
by the quotient space $P_{\mu}=\mathbf{J}^{-1}(\mu) / G_{\mu}$ and with the induced symplectic form $\Omega_{\mu}$ uniquely defined by

$$
\pi_{\mu}^{*} \Omega_{\mu}=i_{\mu}^{*} \Omega
$$

where $\pi_{\mu}: \mathbf{J}^{-1}(\mu) \rightarrow P_{\mu}$ is the projection and $i_{\mu}: \mathbf{J}^{-1}(\mu) \rightarrow P$ is the inclusion. When one chooses $P=T^{*} G$ with $G$ acting by left translation, it was shown in Marsden and Weinstein [42] that the symplectic reduced space $\left(T^{*} G\right)_{\mu}=\mathbf{J}^{-1}(\mu) / G_{\mu}$ is identified via left translation with the coadjoint orbit $\mathcal{O}_{\mu}:=\left\{\operatorname{Ad}_{g^{-1}}^{*} \mu \mid g \in G\right\}=G \cdot \mu$ through $\mu \in \mathfrak{g}^{*}$ and also that the reduced symplectic form coincides with

$$
\Omega_{\mu}\left(\operatorname{ad}_{\xi}^{*} \mu, \operatorname{ad}_{\eta}^{*} \mu\right)=-\langle\mu,[\xi, \eta]\rangle .
$$

On the other hand, it was shown by Marsden and Ratiu [39] that the Poisson structure on $P_{\mu}$ is related with that on $P / G$ in the generalized context of Poisson reduction. Let the Lie group $G$ act freely and properly on a Poisson manifold $P$ by Poisson maps. Suppose that $P / G$ is a smooth manifold which is endowed with the unique Poisson structure such that the canonical projection $\pi: P \rightarrow P / G$ is a Poisson map. For two functions $f, h: P / G \rightarrow \mathbb{R}$, let $F=f \circ \pi$ and $H=h \circ \pi$, so $F$ and $H$ are $f$ and $h$ thought of as $G$-invariant functions on $P$. Then, $\{f, h\}_{P / G}$ is defined by

$$
\{f, h\}_{P / G} \circ \pi=\{F, H\}_{P} .
$$

It is shown that $\{f, h\}_{P / G}$ is well defined by proving that $\{F, H\}_{P}$ is $G$-invariant, which follows from the fact that $F$ and $H$ are $G$-invariant and the group action of $G$ on $P$ consists of Poisson maps. Especially, when $P=T^{*} G$ and the action of $G$ on $T^{*} G$ is by cotangent lift of left (or right) translation of $G$ on itself, the quotient space $\left(T^{*} G\right) / G$ is naturally diffeomorphic to $\mathfrak{g}^{*}$, namely, the dual of the Lie algebra $\mathfrak{g}$ of $G$. Then, the quotient Poisson bracket is given by the plus (or minus) Lie-Poisson bracket as

$$
\{f, h\}_{ \pm}(\mu)=\mp\left\langle\mu,\left[\frac{\delta f}{\delta \mu}, \frac{\delta h}{\delta \mu}\right]\right\rangle,
$$

where $f, h \in \mathcal{F}\left(\mathfrak{g}^{*}\right)$ are arbitrary functions (see Marsden and Weinstein [43]). The minus sign goes with left reduction and the plus sign with right reduction.

As in Marsden and Ratiu [40], it was shown that both Poisson reduction and the symplectic reduction for the case $P=T^{*} G$ in the above are naturally incorporated into the reduction of Hamiltonian systems on a Lie group; namely, in the context of Lie-Poisson reduction, Lie-Poisson equations can be developed as

$$
\dot{\mu}= \pm \operatorname{ad}_{\frac{\delta h}{\delta \mu}}^{*} \mu,
$$

where $h:=H \mid \mathfrak{g}^{*}$ is the reduced Hamiltonian on $\mathfrak{g}^{*}=T_{e}^{*} G \subset T^{*} G$.

On the Lagrangian side, the Lagrangian analogue of Lie-Poisson reduction called Euler-Poincaré reduction can be given in the context of a reduced constrained variational principle (see [40]):

$$
\delta \int_{t_{1}}^{t_{2}} l(\xi(t)) d t=0
$$


with variations restricted to be of the form

$$
\delta \xi=\dot{\eta} \pm[v, \eta],
$$

where $l=L \mid \mathfrak{g}$ is the reduced Lagrangian defined by the restriction of a $G$-invariant Lagrangian $L$ on $T G$ to $\mathfrak{g}=T_{e} G \subset T G$ and $\eta(t)$ is a curve in $\mathfrak{g}$ such that $\eta\left(t_{1}\right)=\eta\left(t_{2}\right)=0$. Then, we can obtain Euler-Poincaré equations as

$$
\frac{d}{d t} \frac{\delta l}{\delta \xi}= \pm \operatorname{ad}_{\xi}^{*} \frac{\delta l}{\delta \xi} .
$$

\section{History and literature}

The development of reduction of Dirac structures is a natural outgrowth of symplectic and Poisson reduction. In the context of Dirac structures, Courant [23] showed that a reduced Dirac structure on $\mathbf{J}^{-1}(\mu) / G_{\mu}$ can be found that is consistent with the Poisson reduction developed by Marsden and Ratiu [39]. In a similar context, reduction of Dirac structures for Hamiltonian systems with symmetry was given in Dorfman [28]. Reduction of implicit Hamiltonian systems was developed by van der Schaft [48], Blankenstein [4], Blankenstein and van der Schaft [5] in a way that is consistent with Courant [23]. Furthermore, singular reduction of Dirac structures was developed by Blankenstein and Ratiu [6] in the context of implicit Hamiltonian systems. In these constructions, it was shown that a reduced Dirac structure fits naturally into the context of symplectic reduction as well as Poisson reduction; if a Dirac structure $D \subset T P \oplus T^{*} P$ on $P$ is the graph of the bundle map $\omega^{\mathrm{b}}: T P \rightarrow T^{*} P$ associated with a symplectic structure $\omega$, then a reduced Dirac structure $D_{\mu}$ on $P_{\mu}=\mathbf{J}^{-1}(\mu) / G_{\mu}$ may be given by the graph of the reduced bundle map associated with the reduced symplectic structure on $P_{\mu}$, while if $D$ is the graph of the bundle map $B^{\sharp}: T^{*} P \rightarrow T P$ associated with a Poisson structure $B$ on $P$, then the reduced Dirac structure can be given by the graph of the bundle map associated with the reduced Poisson structure on $P_{\mu}$.

From the viewpoint of reduced variational principles, it was shown in [18] that the Lie-Poisson equations can be formulated by a reduced constrained variational principle called the Lie-Poisson variational principle, which is a Hamiltonian analogue of the reduced constrained variational principle for the Euler-Poincaré equations. On the other hand, investigations have been lacking on the issue of how both Euler-Poincaré and Lie-Poisson reductions can be linked with reduction of a Dirac structure and with reduction of implicit Lagrangian and Hamiltonian systems. As we mentioned previously, one of our main goals is to fill this gap.

\section{The applied viewpoint}

Needless to say, from application points of view, Euler-Poincaré and Lie-Poisson equations were made essential use in examples such as rigid body dynamics, hydrodynamics, plasma physics, etc (various references may be found in Marsden 
[37], Marsden and Ratiu [40]), as well as in numerical simulations, such as BouRabee and Marsden [11] and references therein. Furthermore, it is worth noting that the Euler-Poincaré equations with nonholonomic constraints (called the EulerPoincaré-Suslov equations) are essential in nonholonomic rigid body mechanics (see, $[29,7])$. In this paper, we will develop an implicit analogue of Euler-Poincaré-Suslov equations in the context of Dirac reduction, together with the development of an implicit Hamiltonian analogue which we call the Lie-Poisson-Suslov equations.

\section{Groupoids and algebroids}

One of other interesting issues relevant to this paper is concerned with Lie algebroids in Lagrangian mechanics, which were introduced by Weinstein [49]. In particular, a Dirac structure on a Lie algebroid was constructed in a way that is dual to a Poisson structure on the dual bundle of the Lie algebroid; Lagrangian reduction was thereby developed in the context of Lie algebroids. In relation with Drinfel'd's theory of Lie bialgebras and Poisson homogeneous spaces, Poisson homogeneous spaces for Poisson groupoids were classified with the help of Dirac structures by Liu, Weinstein and $\mathrm{Xu}$ [36]. A recent theoretical development in Dirac structures may be found in, for instance, Bursztyn, Crainic, Weinstein and Zhu [15], where twisted Dirac structures were investigated in relation with twisted presymplectic groupoids, and the generalized ideas of momentum maps in Dirac geometry called Dirac realizations were developed together with associated reduction procedures in Bursztyn and Crainic [14]. Some relevant works in this direction of reduction of Courant algebroids and the Dirac structures therein can be found in, for instance, $[12,13]$, where the authors mainly focused on the so-called "exact" Courant algebroids.

Outline of the Paper. In Section 2, we give a brief review of induced Dirac structures and their associated implicit Lagrangian systems. In Sextion 3, we show how the reduction of the Hamilton-Pontryagin principle enables us to formulate implicit Euler-Poincaré equations, which may be viewed as an implicit analogue of Euler-Poincaré equations. When a given Lagrangian is regular, via the Legendre transformation, we can also show that implicit Lie-Poisson equations can be developed by the reduction of Hamilton's phase space principle, namely, the Lie-Poisson variational principle. In Section 4, we develop a reduction procedure called LieDirac reduction to investigate the reduction of the canonical Dirac structure $D$ on $T^{*} G$. In this case, the canonical Dirac structure $D \subset T T^{*} G \oplus T^{*} T^{*} G$ can be thought of as the graph of the canonical symplectic structure or the graph of the canonical Poisson structure. Using the left trivializing diffeomorphism $T^{*} G \rightarrow G \times \mathfrak{g}^{*}$, we set up a reduction procedure of the canonical Dirac structure on $T^{*} G \cong G \times \mathfrak{g}^{*}$ by taking quotients by $G$; namely, the canonical Dirac structure can be reduced to a Dirac structure on $\mathfrak{g} \oplus \mathfrak{g}^{*}$ at each point $\mu \in \mathfrak{g}^{*} \cong\left(T^{*} G\right) / G$, which naturally includes the coadjoint orbit symplectic structure $\omega_{\mu}$, the Lie-Poisson structure as well as the reduced canonical symplectic structure on $\mathfrak{g} \oplus \mathfrak{g}^{*}$. In $\S 5$, by using the reduced Dirac 
structure on $\mathfrak{g} \oplus \mathfrak{g}^{*}$, we establish a reduction procedure called Euler-Poincaré-Dirac reduction to obtain implicit Euler-Poincaré equations. In Section 6, when a given Lagrangian is regular, we develop a reduction procedure called Lie-Poisson-Dirac reduction to obtain implicit Lie-Poisson equations in the dual context of EulerPoincaré-Dirac reduction. In Section 7, for the case in which one has a constraint distribution $\Delta_{G} \subset T G$, we develop the reduction procedure for a Dirac structure induced from a nontrivial distribution on $G$ and then we construct the associated Euler-Poincaré-Suslov and Lie-Poisson-Suslov reductions, together with the Suslov problem in nonholonomic mechanics. In Section 10, concluding remarks are given together with future work.

\section{Review of implicit Lagrangian systems}

In this paper, we shall investigate the reduction of a Dirac structure on the cotangent bundle of a Lie group (which is induced from an invariant distribution on a Lie group) and then we shall also explore reduction of associated implicit Lagrangian systems.

Before going into the details of Dirac reduction, we shall briefly review how to define an induced Dirac structure and, given a Lagrangian, an associated implicit Lagrangian system, following Courant [23] and Yoshimura and Marsden [50].

\section{Dirac structures}

We first recall the definition of a Dirac structure on a vector space $V$, say finite dimensional for simplicity. Let $V^{*}$ be the dual space of $V$, and $\langle\cdot, \cdot\rangle$ be the natural paring between $V^{*}$ and $V$. Define the symmetric paring $\langle\langle\cdot, \cdot\rangle\rangle$ on $V \oplus V^{*}$ by

$$
\langle\langle(v, \alpha),(\bar{v}, \bar{\alpha})\rangle\rangle=\langle\alpha, \bar{v}\rangle+\langle\bar{\alpha}, v\rangle,
$$

for $(v, \alpha),(\bar{v}, \bar{\alpha}) \in V \oplus V^{*}$. A Dirac structure on $V$ is a subspace $D \subset V \oplus V^{*}$ such that $D=D^{\perp}$, where $D^{\perp}$ is the orthogonal of $D$ relative to the pairing $\langle\langle\cdot, \cdot\rangle\rangle$.

Now let $P$ be a given manifold and let $T P \oplus T^{*} P$ denote the Whitney sum bundle over $P$, namely, the bundle over the base $P$ and with fiber over the point $x \in P$ equal to $T_{x} P \times T_{x}^{*} P$. In this paper, we shall call a subbundle $D \subset T P \oplus T^{*} P$ a Dirac structure on $P$ when it is a Dirac structure in the sense of vector spaces at each point $x \in P$. A given two-form $\Omega$ on $P$ together with a distribution $\Delta$ on $P$ determines a Dirac structure on $P$ as follows: let $x \in P$, and define

$$
\begin{aligned}
D(x)=\left\{\left(v_{x}, \alpha_{x}\right) \in T_{x} P \times T_{x}^{*} P \mid v_{x}\right. & \in \Delta(x), \text { and } \\
\alpha_{x}\left(w_{x}\right) & \left.=\Omega_{\Delta}\left(v_{x}, w_{x}\right) \text { for all } w_{x} \in \Delta(x)\right\},
\end{aligned}
$$

where $\Omega_{\Delta}$ is the restriction of $\Omega$ to $\Delta$.

We call a Dirac structure $D$ integrable if the condition

$$
\left\langle £_{X_{1}} \alpha_{2}, X_{3}\right\rangle+\left\langle £_{X_{2}} \alpha_{3}, X_{1}\right\rangle+\left\langle £_{X_{3}} \alpha_{1}, X_{2}\right\rangle=0
$$


is satisfied for all pairs of vector fields and one-forms $\left(X_{1}, \alpha_{1}\right),\left(X_{2}, \alpha_{2}\right),\left(X_{3}, \alpha_{3}\right)$ that take values in $D$, where $£_{X}$ denotes the Lie derivative along the vector field $X$ on $P$. In this paper, we are primarily interested in Dirac structures that need not be integrable.

\section{Induced Dirac structures}

One of the most important and interesting Dirac structures in mechanics is one that is induced from nonholonomic kinematic constraints. Such constraints are generally given by a distribution on a configuration manifold.

Let $Q$ be a configuration manifold. Let $T Q$ be the tangent bundle and $T^{*} Q$ be the cotangent bundle. Let $\Delta_{Q} \subset T Q$ be a regular distribution on $Q$ and define a lifted distribution on $T^{*} Q$ by

$$
\Delta_{T^{*} Q}=\left(T \pi_{Q}\right)^{-1}\left(\Delta_{Q}\right) \subset T T^{*} Q,
$$

where $\pi_{Q}: T^{*} Q \rightarrow Q$ is the canonical projection so that its tangent is a map $T \pi_{Q}: T T^{*} Q \rightarrow T Q$. Let $\Omega$ be the canonical two-form on $T^{*} Q$. The induced Dirac structure $D_{\Delta_{Q}}$ on $T^{*} Q$, is the subbundle of $T T^{*} Q \oplus T^{*} T^{*} Q$, whose fiber is given for each $p_{q} \in T^{*} Q$ as

$$
\begin{aligned}
& D_{\Delta_{Q}}\left(p_{q}\right)=\left\{\left(v_{p_{q}}, \alpha_{p_{q}}\right) \in T_{p_{q}} T^{*} Q \times T_{p_{q}}^{*} T^{*} Q \mid v_{p_{q}} \in \Delta_{T^{*} Q}\left(p_{q}\right),\right. \text { and } \\
& \left.\alpha_{p_{q}}\left(w_{p_{q}}\right)=\Omega\left(p_{q}\right)\left(v_{p_{q}}, w_{p_{q}}\right) \text { for all } w_{p_{q}} \in \Delta_{T^{*} Q}\left(p_{q}\right)\right\} .
\end{aligned}
$$

This is, of course, a special instance of the construction (2.1).

\section{Implicit Lagrangian systems}

Let us recall the definition of implicit Lagrangian systems; once again, for further details, see [50].

Let $L: T Q \rightarrow \mathbb{R}$ be a Lagrangian, possibly degenerate. Recall that the differential of $L$ is a map $\mathbf{d} L: T Q \rightarrow T^{*} T Q$, which is locally expressed by using local coordinates $(q, v)$ for $T Q$ as

$$
\mathbf{d} L=\left(q, v, \frac{\partial L}{\partial q}, \frac{\partial L}{\partial v}\right) .
$$

The Dirac differential of the Lagrangian $L: T Q \rightarrow \mathbb{R}$, namely, $\mathfrak{D} L$ is defined by

$$
\mathfrak{D} L=\gamma_{Q} \circ \mathbf{d} L: T Q \rightarrow T^{*} T^{*} Q .
$$

In the above, $\gamma_{Q}: T^{*} T Q \rightarrow T^{*} T^{*} Q$ is the natural diffeomorphism given in coordinates by

$$
(q, \delta q, \delta p, p) \rightarrow(q, p,-\delta p, \delta q),
$$

where $(q, \delta q, \delta p, p)$ are the local coordinates for $T^{*} T Q$ and $(q, p,-\delta p, \delta q)$ for $T^{*} T^{*} Q$. Consequently, one has the following local expression of the Dirac differential 
of $L$ :

$$
\mathfrak{D} L=\left(q, \frac{\partial L}{\partial v},-\frac{\partial L}{\partial q}, v\right),
$$

which is an element of $T^{*} T^{*} Q$ with base point

$$
(q, p)=\left(q, \frac{\partial L}{\partial v}\right)
$$

Let $X: T Q \oplus T^{*} Q \rightarrow T T^{*} Q$ be a partial vector field on $T^{*} Q$; that is, a map that assigns to each point $(q, v, p) \in T Q \oplus T^{*} Q$, a vector in $T T^{*} Q$ at the point $(q, p) \in T^{*} Q$; we write $X$ as

$$
X(q, v, p)=(q, p, \dot{q}, \dot{p}),
$$

where $\dot{q}, \dot{p}$ are understood to be functions of $(q, v, p)$. An implicit Lagrangian system is a triple $\left(L, \Delta_{Q}, X\right)$, which satisfies, for each $v \in \Delta_{Q}(q)$,

$$
(X(q, v, p), \mathfrak{D} L(q, v)) \in D_{\Delta_{Q}}(q, p),
$$

where $X$ is a partial vector field on $T^{*} Q$ defined at points $(q, v, p)$ satisfying $p=\mathbb{F} L(q, v)$ for $v \in \Delta_{Q}$.

\section{Coordinate representations}

The canonical two-form $\Omega$ is given, in coordinates, by

$$
\Omega\left(\left(q, p, u_{1}, \alpha_{1}\right),\left(q, p, u_{2}, \alpha_{2}\right)\right)=\left\langle\alpha_{2}, u_{1}\right\rangle-\left\langle\alpha_{1}, u_{2}\right\rangle,
$$

and the induced Dirac structure may be expressed, in coordinates, by

$$
D_{\Delta_{Q}}(q, p)=\left\{((q, p, \dot{q}, \dot{p}),(q, p, \alpha, w)) \mid \dot{q} \in \Delta(q), \quad w=\dot{q}, \quad \text { and } \alpha+\dot{p} \in \Delta^{\circ}(q)\right\},
$$

where $\Delta^{\circ}(q) \subset T^{*} Q$ is the polar of $\Delta(q)$. Writing $X(q, v, p)=(q, p, \dot{q}, \dot{p})$ and with $p=\partial L / \partial v$, and using the local expressions for the canonical symplectic form and the Dirac differential, the condition for an implicit Lagrangian system $(X, \mathfrak{D} L) \in D_{\Delta_{Q}}$ reads as follows: $p=\partial L / \partial v$ (equality of base points) and

$$
\left\langle-\frac{\partial L}{\partial q}, u\right\rangle+\langle v, \alpha\rangle=\langle\alpha, \dot{q}\rangle-\langle\dot{p}, u\rangle
$$

for all $u \in \Delta(q)$ and all $\alpha$, where $(u, \alpha)$ are the local representatives of a point in $T_{(q, p)} T^{*} Q$. Since this holds for all $u \in \Delta(q)$ and all $\alpha$, we obtain the coordinate representation of an implicit Lagrangian system as

$$
p=\frac{\partial L}{\partial v}, \quad \dot{q}=v \in \Delta(q), \quad \dot{p}-\frac{\partial L}{\partial q} \in \Delta^{\circ}(q) .
$$

Notice that if a partial vector field $X(q, v, p)=(q, p, \dot{q}, \dot{p})$ satisfies the Dirac condition $(X, \mathfrak{D} L) \in D$, then the Legendre transformation $p=\partial L / \partial v$ is consistent with the equality of base points and that the Dirac condition itself implies that $\dot{q}$ is given by the second order condition $\dot{q}=v$. 
In this paper, we primarily focus on the case $Q=G$; namely, our configuration space $Q$ is a Lie group $G$. At first, we shall investigate the reduction of the canonical Dirac structure, and in the case $\Delta_{G}=T G$; namely, there is no constraint imposed. Later, we shall return to the general case in which the Dirac structure is induced from $\Delta_{G} \subset T G$ and we shall develop a reduction method for implicit Lagrangian systems. Then, we shall apply this to the Suslov problem (see Bloch [7], Section 8.6).

\section{The reduced Hamilton-Pontryagin principle}

In this section, we begin by recalling an extended variational principle called the Hamilton-Pontryagin principle, a principle first studied by Livens [35]. It is called the Hamilton-Pontryagin principle because of its close relation with both Hamilton's principle and the Pontryagin principle in control theory (see Pontryagin, Boltyanskiı̌, Gamkrelidze and Mishchenko [45], Sussmann [46]). This principle has as its associated equations, the implicit Euler-Lagrange equations, as was studied in Yoshimura and Marsden [51].

Then, it is shown how the Hamilton-Pontryagin principle on $T G \oplus T^{*} G$ can be reduced, where implicit Euler-Poincaré equations can be obtained by reducing implicit Euler-Lagrange equations. Further, on the Hamiltonian side, it is shown how Hamilton's phase space principle on $T G \oplus T^{*} G$ can be reduced in the context of Lie-Poisson variational principle, where implicit Lie-Poisson equations can be formulated by the reduction of Hamilton's equations.

\section{The Hamilton-Pontryagin principle}

Let $G$ be a Lie group, $T G$ the tangent bundle and $T^{*} G$ the cotangent bundle. Let $g \in G,(g, v) \in T G$ and $(g, p) \in T^{*} G$. Let $(g, v, p)$ be local coordinates for the Pontryagin bundle $T G \oplus T^{*} G$. Let $L: T G \rightarrow \mathbb{R}$ be a left invariant Lagrangian (possibly, degenerate).

Define the associated action integral on the space of curves $(g(t), v(t), p(t)), t_{1} \leq$ $t \leq t_{2}$, in $T G \oplus T^{*} G$ by

$$
\mathfrak{F}(g, v, p)=\int_{t_{1}}^{t_{2}}\{L(g(t), v(t))+\langle p(t), \dot{g}(t)-v(t)\rangle\} d t .
$$

The Hamilton-Pontryagin principle is the condition of stationarity of $\mathfrak{F}$;

$$
\delta \mathfrak{F}=0 .
$$


Explicitly, this principle is given by

$$
\begin{aligned}
\delta \mathfrak{F}(g, v, p) & =\delta \int_{t_{1}}^{t_{2}}\{L(g(t), v(t))+\langle p(t), \dot{g}(t)-v(t)\rangle\} d t \\
& =\int_{t_{1}}^{t_{2}}\left\{(\dot{g}-v) \delta p+\left(-\dot{p}+\frac{\partial L}{\partial g}\right) \delta g+\left(-p+\frac{\partial L}{\partial v}\right) \delta v\right\} d t+\left.p \delta g\right|_{t_{1}} ^{t_{2}} \\
& =0,
\end{aligned}
$$

which is to be satisfied for all $\delta g, \delta v$ and $\delta p$ with appropriate boundary conditions. Keeping the endpoints $g\left(t_{1}\right)$ and $g\left(t_{2}\right)$ of $g(t)$ fixed, we obtain the coordinate expression

$$
p=\frac{\partial L}{\partial v}, \quad \dot{g}=v, \quad \dot{p}=\frac{\partial L}{\partial g},
$$

which we shall call implicit Euler-Lagrange equations on $T G \oplus T^{*} G$.

Note that the implicit Euler-Lagrange equations include the Euler-Lagrange equations $\dot{p}=\partial L / \partial g$, the Legendre transformation $p=\partial L / \partial v$ and the second-order condition $\dot{g}=v$.

\section{Reduction of the Hamilton-Pontryagin principle}

The group $G$ acts on curves in the action integral $\mathfrak{F}$, in the Hamilton-Pontryagin principle, by simultaneously left translating on each factor by the left-action and the tangent and cotangent lifts. Explicitly, the action of an element $h \in G$ is given on a curve $g(t) \in G, v(t) \in T_{g(t)} G$ and $p(t) \in T_{g(t)}^{*} G$, by

$$
h \cdot(g(t), v(t), p(t))=\left(h g(t), T_{g(t)} L_{h} \cdot v(t), T_{h g(t)}^{*} L_{h^{-1}} \cdot p(t)\right),
$$

where $T_{g(t)} L_{h}: T_{g(t)} G \rightarrow T_{h g(t)} G$ is the tangent of the left translation map $L_{h}: G \rightarrow$ $G ; g(t) \mapsto h g(t)$ at the point $g(t)$ and $T_{h g(t)}^{*} L_{h^{-1}}: T_{g(t)}^{*} G \rightarrow T_{h g(t)}^{*} G$ is the dual of the map $T_{h g(t)} L_{h^{-1}}: T_{h g(t)} G \rightarrow T_{g(t)} G$. The action integral $\mathfrak{F}$ is invariant under the action of $G$ since $L$ is $G$-invariant and one easily checks that $\langle p(t), \dot{g}(t)-v(t)\rangle$ is also $G$-invariant.

By the following diffeomorphisms, the cotangent bundle $T^{*} G$ is diffeomorphic to $G \times \mathfrak{g}^{*}$ as

$$
\bar{\lambda}: T^{*} G \rightarrow G \times \mathfrak{g}^{*}: \quad \bar{\lambda}\left(p_{g}\right)=\left(g, T_{e}^{*} L_{g}(p)\right),
$$

while the tangent bundle $T G$ is diffeomorphic to $G \times \mathfrak{g}$ by

$$
\lambda: T G \rightarrow G \times \mathfrak{g}: \quad \lambda\left(v_{g}\right)=\left(g, T_{g} L_{g^{-1}}(v)\right) .
$$

Similarly, the Pontryagin bundle $T G \oplus T^{*} G$ is naturally diffeomorphic to its left trivialization as $G \times\left(\mathfrak{g} \oplus \mathfrak{g}^{*}\right)$.

Let $L$ be a left-invariant Lagrangian on $T G$ and let $l: \mathfrak{g} \rightarrow \mathbb{R}$ be the reduced Lagrangian given by $l:=L \mid \mathfrak{g}$, where we regard $\mathfrak{g}=T_{e} G \subset T G$. Thus, by left 
invariance, we have $L(g, \dot{g})=l(\xi)$, where $\xi=g^{-1} \dot{g}$ and the notation $g^{-1} \dot{g}$ stands for $T_{g} L_{g^{-1}} \dot{g}$.

The reduction of the Hamilton-Pontryagin principle is given by

$$
\delta \int_{t_{1}}^{t_{2}}\{l(\eta(t))+\langle\mu(t), \xi(t)-\eta(t)\rangle\} d t=0,
$$

where, again, $\xi=g^{-1} \dot{g}$. Taking variations in (3.3) yields

$$
\begin{aligned}
\int_{t_{1}}^{t_{2}} & \left\{\frac{\delta l}{\delta \eta} \delta \eta+\delta \mu \cdot(\xi-\eta)+\mu \cdot(\delta \xi-\delta \eta)\right\} d t \\
& =\int_{t_{1}}^{t_{2}}\left\{\left(\frac{\delta l}{\delta \eta}-\mu\right) \delta \eta+\delta \mu \cdot(\xi-\eta)+\mu \cdot \delta \xi\right\} d t \\
& =\int_{t_{1}}^{t_{2}}\left\{\left(\frac{\delta l}{\delta \eta}-\mu\right) \delta \eta+\delta \mu \cdot(\xi-\eta)+\mu \cdot\left(\dot{\zeta}+\operatorname{ad}_{\xi} \zeta\right)\right\} d t \\
& =\int_{t_{1}}^{t_{2}}\left\{\left(\frac{\delta l}{\delta \eta}-\mu\right) \delta \eta+\delta \mu \cdot(\xi-\eta)+\left(-\dot{\mu}+\operatorname{ad}_{\xi}^{*} \mu\right) \cdot \zeta\right\} d t+[\mu \cdot \zeta]_{t_{1}}^{t_{2}},
\end{aligned}
$$

where we utilize the relation $\xi=T_{g} L_{g^{-1}} \dot{g}$ and the variation of $\xi$ is computed exactly as in Euler-Poincaré theory to be given by $\delta \xi(t)=\dot{\zeta}(t)+[\xi(t), \zeta(t)]$, where $\zeta=T_{g} L_{g^{-1}} \delta g$, so that $\zeta(t)$ is an arbitrary curve in $\mathfrak{g}$ satisfying $\zeta\left(t_{1}\right)=\zeta\left(t_{2}\right)=0$. Then, Eq. (3.4) vanishes for any $\delta \eta \in \mathfrak{g}, \zeta \in \mathfrak{g}$ and $\delta \mu \in \mathfrak{g}^{*}$ if and only if

$$
\mu=\frac{\delta l}{\delta \eta}, \quad \xi=\eta, \quad \dot{\mu}=\operatorname{ad}_{\xi}^{*} \mu .
$$

We shall call the set of Eqs. (3.5) implicit Euler-Poincaré equations on $\mathfrak{g} \oplus \mathfrak{g}^{*}$, which may be thought of as the reduction of implicit Euler-Lagrange equations on $T G \oplus T^{*} G$ in Eq. (3.2). We remark that in this reduced variational principle for implicit Euler-Poincaré equations, the reduced Lagrangian $l$ on $\mathfrak{g}$ may be degenerate.

\section{Hamilton's principle in phase space}

For the case in which a given Lagrangian $L$ on $T G$ is regular and left invariant, we can define a Hamiltonian on $T^{*} G$ by the Legendre transformation. Recall that we define the energy $E: T G \rightarrow \mathbb{R}$ by $E(g, v)=A(g, v)-L(g, v)$, where $A(v)=\mathbb{F} L(v) \cdot v$ is the action of $L(v)$ for $v \in T_{g} G$. Then, we define the Hamiltonian $H$ on $T^{*} G$ as

$$
H=E \circ(\mathbb{F} L)^{-1},
$$

where $\mathbb{F} L: T G \rightarrow T^{*} G$. It is easily checked that $H$ is also left invariant (see Marsden and Ratiu [40]).

Define the phase space action integral on the space of curves $(g(t), p(t)), t_{1} \leq$ 
$t \leq t_{2}$ in $T^{*} G$ by

$$
\mathfrak{S}(g(t), p(t))=\int_{t_{1}}^{t_{2}}\{\langle p(t), \dot{g}(t)\rangle-H(g(t), p(t))\} d t .
$$

Hamilton's phase space principle states that this action is stationary with respect to varying curves $(q, p)$ :

$$
\delta \mathfrak{S}=0
$$

again, with appropriate boundary conditions on the variations. Explicitly, the variation is

$$
\begin{aligned}
\delta \mathfrak{S}(g, p) & =\delta \int_{t_{1}}^{t_{2}}\{\langle p(t), \dot{g}(t)\rangle-H(g(t), p(t))\} d t \\
& =\int_{t_{1}}^{t_{2}}\left\{\left(-\dot{p}-\frac{\partial H}{\partial g}\right) \delta g+\left(-\dot{g}+\frac{\partial H}{\partial p}\right) \delta p\right\} d t+\left.p \delta g\right|_{t_{1}} ^{t_{2}} \\
& =0
\end{aligned}
$$

which is satisfied for all $\delta g$ and $\delta p$. Keeping the endpoints $g\left(t_{1}\right)$ and $g\left(t_{2}\right)$ of $g(t)$ fixed, we obtain Hamilton's equations on $T^{*} G$ as

$$
\dot{g}=\frac{\partial H}{\partial p}, \quad \dot{p}=-\frac{\partial H}{\partial g} .
$$

One can use the Hamilton-Pontryagin principle to derive Hamilton's phase space principle in the regular case as follows. In the principle (3.1), one first takes the variation with respect to $v$; notice that (as in Yoshimura and Marsden [51]) the Hamiltonian is given by making the quantity $\langle p, v\rangle-L(q, v)$ stationary in $v$. Then using the remaining variations in $q$ and $p$, the principle (3.1) becomes (3.6).

\section{The Lie-Poisson variational principle}

The group $G$ acts on curves in phase space by simultaneously left translating each of $q$ and $p$ by the left-action. Then, it is easily checked that the action integral is invariant under this action of $G$ since we assume the invariance of $H$.

Since $H$ is left invariant on $T^{*} G$, it defines a reduced Hamiltonian $h: \mathfrak{g}^{*} \rightarrow \mathbb{R}$. Clearly, the reduction of Hamilton's phase space principle is given by

$$
\delta \int_{t_{1}}^{t_{2}}\{\mu(t) \cdot \xi(t)-h(\mu(t))\} \quad d t=0,
$$

where $\xi=g^{-1} \dot{g}$, as in the reduced Hamilton-Pontryagin principle. This reduced principle is called as Lie-Poisson variational principle; see Cendra, Marsden, Pekarsky and Ratiu [18]. 
Taking variations the curve $(\xi(t), \mu(t)) \in \mathfrak{g} \oplus \mathfrak{g}^{*}$ of Eq. (3.8) yields

$$
\begin{aligned}
\int_{t_{1}}^{t_{2}}(\delta \mu \cdot \xi+\mu & \left.\cdot \delta \xi-\frac{\delta h}{\delta \mu} \delta \mu\right) d t=\int_{t_{1}}^{t_{2}}\left\{\left(\xi-\frac{\delta h}{\delta \mu}\right) \delta \mu+\mu \cdot \delta \xi\right\} d t \\
= & \int_{t_{1}}^{t_{2}}\left\{\left(\xi-\frac{\delta h}{\delta \mu}\right) \delta \mu+\mu \cdot(\dot{\eta}+[\xi, \eta])\right\} d t \\
& =\int_{t_{1}}^{t_{2}}\left\{\left(\xi-\frac{\delta h}{\delta \mu}\right) \delta \mu+\mu \cdot\left(\dot{\eta}+\operatorname{ad}_{\xi} \eta\right)\right\} d t \\
& =\int_{t_{1}}^{t_{2}}\left\{\left(\xi-\frac{\delta h}{\delta \mu}\right) \delta \mu+\left(-\dot{\mu}+\operatorname{ad}_{\xi}^{*} \mu\right) \cdot \eta\right\} d t+[\mu \cdot \eta]_{t_{1}}^{t_{2}} .
\end{aligned}
$$

In the above, the variation of $\xi=T_{g} L_{g^{-1}} \cdot \dot{g}$ is given by $\delta \xi=\dot{\eta}+[\xi, \eta]$, where $\eta(t)=T_{g} L_{g^{-1}} \delta g(t)$ satisfies the boundary conditions $\eta\left(t_{1}\right)=\eta\left(t_{2}\right)=0$. Therefore, Eq. (3.9) vanishes for any $\delta \mu \in \mathfrak{g}^{*}$ and $\eta \in \mathfrak{g}$, if and only if

$$
\xi=\frac{\delta h}{\delta \mu}, \quad \dot{\mu}=\operatorname{ad}_{\xi}^{*} \mu .
$$

We shall call the set of equations in Eq. (3.10) implicit Lie-Poisson equations on $\mathfrak{g} \oplus \mathfrak{g}^{*}$, which may be the reduction of Hamilton's equations on $T^{*} G$ in Eq. (3.7), which is consistent with the Lie-Poisson variational principle.

\section{Lie-Dirac reduction}

In this section, we develop a reduction theory that we shall call Lie-Dirac reduction, where reduction of the canonical Dirac structure on $T^{*} G$ is given by a $\mu$-dependent Dirac structure on $\mathfrak{g} \oplus \mathfrak{g}^{*}$.

\section{The canonical Dirac structure on $T^{*} G$}

Recall that a distribution $\Delta_{G}$ induces a Dirac structure $D_{\Delta_{G}}$ on $T^{*} G$. In the case when $\Delta_{G}=T G$, we get the canonical Dirac structure on the cotangent bundle $T^{*} G$ and denote it simply by $D$. As in the general case, $D$ can be defined by either of the canonical symplectic or the canonical Poisson structures; namely, by the graph of the bundle map $\Omega^{\mathrm{b}}: T T^{*} G \rightarrow T^{*} T^{*} G$ associated with the canonical symplectic structure $\Omega$ on $T^{*} G$ or by the graph of the bundle map $B^{\sharp}: T^{*} T^{*} G \rightarrow T T^{*} G$ associated with the canonical Poisson structure $B$ on $T^{*} G$.

Using the canonical symplectic structure, the canonical Dirac structure is given by, for each $p_{g} \in T^{*} G$,

$$
\begin{array}{r}
D\left(p_{g}\right)=\left\{\left(v_{p_{g}}, \alpha_{p_{g}}\right) \in T_{p_{g}} T^{*} G \times T_{p_{g}}^{*} T^{*} G \mid \alpha_{p_{g}}\left(v_{p_{g}}\right)=\Omega\left(p_{g}\right)\left(v_{p_{g}}, w_{p_{g}}\right)\right. \\
\text { for all } \left.w_{p_{g}} \in T_{p_{g}} T^{*} G\right\} .
\end{array}
$$


In local coordinates, we write the canonical symplectic form as

$$
\Omega\left(\left(g, p, u_{1}, \alpha_{1}\right),\left(g, p, u_{2}, \alpha_{2}\right)\right)=\left\langle\alpha_{2}, u_{1}\right\rangle-\left\langle\alpha_{1}, u_{2}\right\rangle .
$$

\section{The Dirac structure on $G \times \mathfrak{g}^{*}$}

Using the left trivializing diffeomorphism $\bar{\lambda}: T^{*} G \rightarrow G \times \mathfrak{g}^{*}$, we define the canonical one-form $\theta$ on $G \times \mathfrak{g}^{*}$ and the canonical two-form $\omega$ on $G \times \mathfrak{g}^{*}$ as

$$
\theta=\bar{\lambda}_{*} \Theta \quad \text { and } \quad \omega=\bar{\lambda}_{*} \Omega,
$$

where $\Theta$ is the canonical one-form on $T^{*} G$ and $\Omega=-\mathbf{d} \Theta$. Then, the canonical one-form $\theta$ on $G \times \mathfrak{g}^{*}$ is locally represented at each $(g, \mu) \in G \times \mathfrak{g}^{*}$ and $(v, \rho) \in$ $T_{(g, \mu)}\left(G \times \mathfrak{g}^{*}\right) \cong T_{g} G \times \mathfrak{g}^{*}$, as

$$
\theta(g, \mu) \cdot(v, \rho)=\mu\left(T_{g} L_{g^{-1}} v\right),
$$

and hence the symplectic structure $\omega=-\mathbf{d} \theta$ on $G \times \mathfrak{g}^{*}$ at each point $(g, \mu) \in G \times \mathfrak{g}^{*}$, is given by

$$
\begin{aligned}
& \omega(g, \mu)((v, \rho),(w, \sigma)) \\
& =\left\langle\sigma, T_{g} L_{g^{-1}} v\right\rangle-\left\langle\rho, T_{g} L_{g^{-1}} w\right\rangle+\left\langle\mu,\left[T_{g} L_{g^{-1}} v, T_{g} L_{g^{-1}} w\right]\right\rangle,
\end{aligned}
$$

where $(w, \sigma) \in T_{(g, \mu)}\left(G \times \mathfrak{g}^{*}\right) \cong T_{g} G \times \mathfrak{g}^{*}$ and we utilized the formula (see Abraham, Marsden, and Ratiu [2])

$$
\omega(X, Y)=-\mathbf{d} \theta(X, Y)=-X[\theta(Y)]+Y[\theta(X)]+\theta([X, Y]),
$$

where $X(g, \mu)=(v, \rho)$ and $Y(g, \mu)=(w, \sigma)$.

Using the diffeomorphism $\bar{\lambda}: T^{*} G \rightarrow G \times \mathfrak{g}^{*}$, we can naturally define the Dirac structure $\mathcal{D}$ on $G \times \mathfrak{g}^{*}$, which is locally represented by, for each $(g, \mu) \in G \times \mathfrak{g}^{*}$,

$$
\begin{aligned}
& \mathcal{D}(g, \mu)=\left\{((v, \rho),(\beta, \eta)) \in\left(T_{g} G \times \mathfrak{g}^{*}\right) \times\left(T_{g}^{*} G \times \mathfrak{g}\right) \mid\right. \\
& \left.\langle\beta, w\rangle+\langle\sigma, \eta\rangle=\omega(g, \mu)((v, \rho),(w, \sigma)) \text { for all }(w, \sigma) \in T_{g} G \times \mathfrak{g}^{*}\right\} .
\end{aligned}
$$

\section{Dirac maps}

We will show that the Dirac structure $\mathcal{D}$ on $G \times \mathfrak{g}^{*}$ can also be obtained directly by using Dirac maps (see, $[16,15])$.

Let $D_{V}$ and $D_{W}$ be Dirac structures on vector spaces $V$ and $W$. A linear map $\psi: V \rightarrow W$ is a (forward) Dirac map if $D_{V}$ and $D_{W}$ are related by

$$
D_{W}=\left\{(\psi(x), \eta) \mid x \in V, \quad \eta \in W^{*}, \quad\left(x, \psi^{*}(\eta)\right) \in D_{V}\right\},
$$

where $\psi^{*}: W^{*} \rightarrow V^{*}$ is the dual of $\psi$, and we write

$$
D_{W}=\psi_{*} D_{V} .
$$


For the case of manifolds, let $M$ and $N$ be manifolds and let $D_{M}$ and $D_{N}$ be Dirac structures on $M$ and $N$, respectively. A smooth map $\psi: M \rightarrow N$ is called a (forward) Dirac map if, for all $x \in M$,

$$
\begin{aligned}
D_{N}(\psi(x))=\left\{\left(T_{x} \psi\left(w_{x}\right), \alpha_{\psi(x)}\right) \mid w_{x} \in T_{x} M, \quad \alpha_{\psi(x)} \in T_{\psi(x)}^{*} N,\right. & \left.\left(w_{x},(T \psi)^{*}\left(\alpha_{\psi(x)}\right)\right) \in D_{M}(x)\right\},
\end{aligned}
$$

where $T_{x} \psi: T_{x} M \rightarrow T_{\psi(x)} N$ is the tangent map of $\psi: M \rightarrow N$ at $x \in M$ and we write the relationship between the two Dirac structures as

$$
D_{N}=\psi_{*} D_{M} .
$$

Then, the Dirac structure $\mathcal{D}$ on $G \times \mathfrak{g}^{*}$ can be defined using the Dirac map $\bar{\lambda}: T^{*} G \rightarrow G \times \mathfrak{g}^{*} ;$ that is, we have

$$
\mathcal{D}=\bar{\lambda}_{*} D,
$$

which is given by, for each $p_{g} \in T^{*} G$,

$$
\begin{array}{r}
\mathcal{D}\left(\bar{\lambda}\left(p_{g}\right)\right)=\left\{\left(T_{p_{g}} \bar{\lambda}\left(w_{p_{g}}\right), \alpha_{\bar{\lambda}\left(p_{g}\right)}\right) \mid w_{p_{g}} \in T_{p_{g}} T^{*} G, \quad \alpha_{\bar{\lambda}\left(p_{g}\right)} \in T_{\bar{\lambda}\left(p_{g}\right)}^{*}\left(G \times \mathfrak{g}^{*}\right),\right. \\
\left.\left(w_{p_{g}},(T \bar{\lambda})^{*}\left(\alpha_{\bar{\lambda}\left(p_{g}\right)}\right)\right) \in D\left(p_{g}\right)\right\},
\end{array}
$$

where $\bar{\lambda}\left(p_{g}\right)=(g, \mu) \in G \times \mathfrak{g}^{*}$.

Thus, we regard the Dirac structure $\mathcal{D}$ on $G \times \mathfrak{g}^{*}$ as the left trivialized expression for the canonical Dirac structure $D$ on $T^{*} G$.

\section{Invariance of the canonical Dirac structure}

Let us first recall the natural definition of invariant Dirac structures (see, for instance, $[28,36,5])$.

Let $P$ be a manifold and $D$ be a Dirac structure on $P$ with a Lie group $G$ acting on $P$. We denote this action by $\Phi: G \times P \rightarrow P$ and the action of a group element $h \in G$ on a point $x \in P$ by $h \cdot x=\Phi(h, x)=\Phi_{h}(x)$ for $h \in G$ and $x \in P$, so that $\Phi_{h}: P \rightarrow P$. Then, a Dirac structure $D \subset T P \oplus T^{*} P$ is $G$-invariant if

$$
\left(\Phi_{h^{*}} X,\left(\Phi_{h}^{*}\right)^{-1} \alpha\right) \in D
$$

for all $h \in G$ and $(X, \alpha) \in D$.

Next, we show that the Dirac structure in Eq. (4.3) is $G$-invariant, where $G$ acts on the first factor by left multiplication and it acts trivially on the second factor. To do this, let $\Lambda: G \times\left(G \times \mathfrak{g}^{*}\right) \rightarrow G \times \mathfrak{g}^{*}$ denote the $G$-action on $G \times \mathfrak{g}^{*}$, so that holding $g$ fixed, $\Lambda_{g}: G \times \mathfrak{g}^{*} \rightarrow G \times \mathfrak{g}^{*}$ is given by

$$
\Lambda_{h}(g, \mu)=(h g, \mu),
$$

where $h, g \in G$ and $\mu \in \mathfrak{g}^{*}$. Since the action on $T^{*} G$ is canonical, the corresponding symplectic structure $\omega$ is $G$-invariant as well; that is,

$$
\Lambda_{h}^{*} \omega=\omega,
$$


for all $h \in G$. It is easily verified that the Dirac structure $\mathcal{D}$ on $G \times \mathfrak{g}^{*}$ is $G$-invariant as

$$
\left(\Lambda_{h^{*}} X,\left(\Lambda_{h}^{*}\right)^{-1} \alpha\right) \in \mathcal{D}
$$

for all $(X, \alpha) \in \mathcal{D}$.

In fact, in view of Eq. (4.3), the left-invariance of the Dirac structure in the above can be restated as

$$
\mathcal{D}(h g, \mu)=\mathcal{D}(g, \mu),
$$

which holds for all $h, g \in G$ and $\mu \in \mathfrak{g}^{*}$, where

$$
\begin{array}{r}
\mathcal{D}(h g, \mu)=\left\{\left(\left(T_{g} L_{h} v, \rho\right),\left(T_{h g}^{*} L_{h^{-1}} \beta, \eta\right)\right) \in\left(T_{h g} G \times \mathfrak{g}^{*}\right) \times\left(T_{h g}^{*} G \times \mathfrak{g}\right) \mid\right. \\
\left\langle T_{h g}^{*} L_{h^{-1}} \beta, T_{g} L_{h} w\right\rangle+\langle\sigma, \eta\rangle=\omega(h g, \mu)\left(\left(T_{g} L_{h} v, \rho\right),\left(T_{g} L_{h} w, \sigma\right)\right) \\
\left.\quad \text { for all }\left(T_{g} L_{h} w, \sigma\right) \in T_{h g} G \times \mathfrak{g}^{*}\right\} .
\end{array}
$$

\section{The quotient space of the Pontryagin bundle}

Using the left trivialization diffeomorphisms $\bar{\lambda}: T^{*} G \rightarrow G \times \mathfrak{g}^{*}$ and $\lambda: T G \rightarrow G \times$ $\mathfrak{g}$, and the fact that $\mathfrak{g} \cong T_{e} G$ and $\mathfrak{g}^{*} \cong T_{e}^{*} G$, the Pontryagin Bundle $T T^{*} G \oplus T^{*} T^{*} G$ is trivialized as follows:

$$
\begin{aligned}
T T^{*} G \oplus T^{*} T^{*} G & \cong T\left(G \times \mathfrak{g}^{*}\right) \oplus T^{*}\left(G \times \mathfrak{g}^{*}\right) \\
& \cong\left(G \times \mathfrak{g}^{*}\right) \times\left(V \oplus V^{*}\right),
\end{aligned}
$$

where we set $V=\mathfrak{g} \oplus \mathfrak{g}^{*}$ and $V^{*}=\mathfrak{g}^{*} \oplus \mathfrak{g}$ and identify $\left(\mathfrak{g}^{*}\right)^{*}$ with $\mathfrak{g}$. Thus, the Pontryagin bundle $T T^{*} G \oplus T^{*} T^{*} G$ is naturally diffeomorphic to $\left(G \times \mathfrak{g}^{*}\right) \times\left(V \oplus V^{*}\right)$

The action of an element $h \in G$ on an element $(g, \mu, \xi, \rho, \nu, \eta) \in\left(G \times \mathfrak{g}^{*}\right) \times$ $\left(V \oplus V^{*}\right)$ is only on the first component; that is,

$$
h \cdot(g, \mu, \xi, \rho, \nu, \eta)=(h \cdot g, \mu, \xi, \rho, v, \eta) .
$$

Thus, the quotient of the Pontryagin bundle $T T^{*} G \oplus T^{*} T^{*} G$ by the action of $G$ is given by

$$
\left(T T^{*} G \oplus T^{*} T^{*} G\right) / G \cong \mathfrak{g}^{*} \times\left(V \oplus V^{*}\right) .
$$

\section{Lie-Dirac reduction}

Because of the $G$-invariance of $\mathcal{D}$ and the identification in Eq. (4.4), the canonical Dirac structure on $G \times \mathfrak{g}^{*}$

$$
\mathcal{D} \subset T\left(G \times \mathfrak{g}^{*}\right) \oplus T^{*}\left(G \times \mathfrak{g}^{*}\right)
$$

may be identified with

$$
\mathcal{D} \subset\left(G \times \mathfrak{g}^{*}\right) \times\left(V \oplus V^{*}\right),
$$

which is uniquely determined by its value at the identity, namely,

$$
\begin{aligned}
\mathcal{D}(e, \mu)=\{(\xi, \rho),(\nu, \eta)) \in V \oplus V^{*} \mid & \\
& \langle\nu, \zeta\rangle+\langle\sigma, \eta\rangle=\omega(e, \mu)((\xi, \rho),(\zeta, \sigma)) \text { for all }(\zeta, \sigma) \in V\} .
\end{aligned}
$$


In view of the invariance of the Dirac structure, it induces, for each $\mu \in \mathfrak{g}^{*}$, a structure, which we denote by $\mathcal{D}_{\mu}^{/ G} \subset V \oplus V^{*}$ on the quotient space. From (4.5) we see that

$$
\begin{aligned}
\mathcal{D}_{\mu}^{\prime G}=\{((\xi, \rho),(\nu, \eta)) & \in V \oplus V^{*} \mid \\
& \left.\langle\nu, \zeta\rangle+\langle\sigma, \eta\rangle=\omega_{\mu}^{/ G}((\xi, \rho),(\zeta, \sigma)) \text { for all }(\zeta, \sigma) \in V\right\},
\end{aligned}
$$

where $\omega_{\mu}^{/ G}$ is the $\mu$-dependent bilinear form on $V=\mathfrak{g} \oplus \mathfrak{g}^{*}$, which, in view of (4.2), is given by

$$
\omega_{\mu}^{\prime G}((\xi, \rho),(\zeta, \sigma))=\langle\sigma, \xi\rangle-\langle\rho, \zeta\rangle+\langle\mu,[\xi, \zeta]\rangle .
$$

Notice that $\omega_{\mu}^{/ G}$ is in fact a symplectic form on $V$ because it is skew symmetric and, as is easily checked, non-degenerate.

Proposition 4.1. For fixed $\mu \in \mathfrak{g}^{*}, \mathcal{D}_{\mu}^{/ G}$ in Eq. (4.6) is given by

$$
\mathcal{D}_{\mu}^{\prime G}=\left\{((\xi, \rho),(\nu, \xi)) \in V \oplus V^{*} \mid v+\rho=\operatorname{ad}_{\xi}^{*} \mu\right\} .
$$

Proof: Suppose that the condition (4.6) holds, namely, that

$$
\langle\nu, \zeta\rangle+\langle\sigma, \eta\rangle=\omega_{\mu}^{/ G}((\xi, \rho),(\zeta, \sigma))
$$

for all $(\zeta, \sigma) \in V$. First, using (4.7) and setting $\sigma=0$, gives

$$
\langle v+\rho, \zeta\rangle=\left\langle\mu, \operatorname{ad}_{\xi} \zeta\right\rangle \quad \text { for all } \zeta \in \mathfrak{g} .
$$

It follows that $v+\rho=\operatorname{ad}_{\xi}^{*} \mu$. Second, set $\zeta=0$ and then

$$
\langle\sigma, \eta-\xi\rangle=0 \quad \text { for all } \sigma \in \mathfrak{g}^{*} .
$$

Therefore, $\eta=\xi$ and so (4.8) holds. The converse is shown in the same way.

\section{The Dirac structure on $V=\mathfrak{g} \oplus \mathfrak{g}^{*}$}

We have the following important theorem showing that the natural reduction of the standard Dirac structure on $T^{*} G$ gives a $\mu$-dependent Dirac structure on $V=\mathfrak{g} \oplus \mathfrak{g}^{*}$.

THEOREM 4.2. For fixed $\mu \in \mathfrak{g}^{*}, \mathcal{D}_{\mu}^{/ G}$ given by (4.6), or equivalently, (4.8), is a Dirac structure on $V=\mathfrak{g} \oplus \mathfrak{g}^{*}$.

Proof: One proof is simply to note that for fixed $\mu$, Eq. (4.6) is a special case of the construction of a Dirac structure given by Eq. (2.1) on the symplectic manifold $P=V$, with symplectic form $\omega_{\mu}^{/ G}$ and with $\Delta=V$, the whole space.

We shall give another direct proof of this theorem as follows. Noting that the symmetric paring on $V \oplus V^{*}$ is given by

$$
\langle\langle((w, \gamma),(\delta, u)), \quad((\xi, \rho),(\nu, \xi))\rangle=\langle v, w\rangle+\langle\gamma, \xi\rangle+\langle\delta, \xi\rangle+\langle\rho, u\rangle,
$$


the orthogonal space of $\mathcal{D}_{\mu}^{/ G}$ is given, for each $\mu \in \mathfrak{g}^{*}$, by

$$
\begin{aligned}
&\left(\mathcal{D}_{\mu}^{/ G}\right)^{\perp}=\{((w, \gamma),(\delta, u)) \in V \oplus V^{*} \mid \\
&\left.\langle\nu, w\rangle+\langle\gamma, \xi\rangle+\langle\delta, \xi\rangle+\langle\rho, u\rangle=0, \quad v+\rho=\operatorname{ad}_{\xi}^{*} \mu\right\} .
\end{aligned}
$$

What we want to prove is that $\mathcal{D}_{\mu}^{/ G}=\left(\mathcal{D}_{\mu}^{/ G}\right)^{\perp}$. Let us first check that $\mathcal{D}_{\mu}^{/ G} \subset\left(\mathcal{D}_{\mu}^{/ G}\right)^{\perp}$. Let $((w, \gamma),(\delta, w)) \in \mathcal{D}_{\mu}^{/ G}$, where $\gamma+\delta=\operatorname{ad}_{w}^{*} \mu$. Since $v+\rho=\operatorname{ad}_{\xi}^{*} \mu$, we have

$$
\begin{aligned}
\langle\nu, w\rangle+\langle\gamma, \xi\rangle+\langle\delta, \xi\rangle+\langle\rho, w\rangle & =\langle\nu+\rho, w\rangle+\langle\gamma+\delta, \xi\rangle \\
& =\left\langle\operatorname{ad}_{\xi}^{*} \mu, w\right\rangle+\left\langle\operatorname{ad}_{w}^{*} \mu, \xi\right\rangle \\
& =\left\langle\mu, \operatorname{ad}_{\xi} w\right\rangle+\left\langle\mu, \operatorname{ad}_{w} \xi\right\rangle=0 .
\end{aligned}
$$

Therefore, $\mathcal{D}_{\mu}^{/ G} \subset\left(\mathcal{D}_{\mu}^{/ G}\right)^{\perp}$.

Conversely, let us check that $\left(\mathcal{D}_{\mu}^{/ G}\right)^{\perp} \subset \mathcal{D}_{\mu}^{/ G}$. Let $((w, \gamma),(\delta, u)) \in\left(\mathcal{D}_{\mu}^{/ G}\right)^{\perp}$. Setting $\xi=0$ and noting $v+\rho=\operatorname{ad}_{\xi}^{*} \mu$, one has

$$
\langle v, w\rangle+\langle\rho, u\rangle=\left\langle\operatorname{ad}_{\xi}^{*} \mu-\rho, w\right\rangle+\langle\rho, u\rangle=\langle\rho, u-w\rangle=0
$$

for all $\rho$. Therefore, $u=w$. Next, letting $\xi$ be arbitrary and noting $w=u$, one has

$$
\begin{aligned}
\langle\nu+\rho, w\rangle+\langle\gamma+\delta, \xi\rangle & =\left\langle\operatorname{ad}_{\xi}^{*} \mu, w\right\rangle+\langle\gamma+\delta, \xi\rangle \\
& =\left\langle\mu, \operatorname{ad}_{\xi} w\right\rangle+\langle\gamma+\delta, \xi\rangle \\
& =-\left\langle\mu, \operatorname{ad}_{w} \xi\right\rangle+\langle\gamma+\delta, \xi\rangle \\
& =\left\langle-\operatorname{ad}_{w}^{*} \mu+\gamma+\delta, \xi\right\rangle=0
\end{aligned}
$$

for all $\xi$. So, we have $\gamma+\delta=\operatorname{ad}_{w}^{*} \mu$. Hence $\left(\mathcal{D}_{\mu}^{/ G}\right)^{\perp} \subset \mathcal{D}_{\mu}^{/ G}$.

Finally, $\left(\mathcal{D}_{\mu}^{/ G}\right)^{\perp}=\mathcal{D}_{\mu}^{/ G}$ and thus, $\mathcal{D}_{\mu}^{/ G}$ is a Dirac structure on $V=\mathfrak{g} \oplus \mathfrak{g}^{*}$.

REMARKS. Note that the reduced symplectic structure $\omega_{\mu}^{/ G}$ includes the canonical symplectic structure on $V=\mathfrak{g} \oplus \mathfrak{g}^{*}$ as well as the Lie-Poisson structure on $\mathfrak{g}^{*}$. This type of symplectic structure is related to symplectic and Poisson structures with cocycles, as in Cendra, Marsden, and Ratiu [21].

It is possible that the reduced Dirac structure $\mathcal{D}_{\mu}^{/ G}$ on $V=\mathfrak{g} \oplus \mathfrak{g}^{*}$ has some connection with Drinfel'd's theory that uses the double $\mathfrak{g} \oplus \mathfrak{g}^{*}$ of the Lie bialgebra $\left(\mathfrak{g}, \mathfrak{g}^{*}\right.$ ) (see Drinfel'd [26]), where Lie bialgebra structures are developed for Hamiltonian structures on Lie groups.

REMARKS. One of the reviewers of this paper made some helpful comments on the relationship between Lie-Dirac reduction of the present paper and the reduction of "Courant algebroids". The point is that the Lie-Dirac reduction procedure, including the case with a given distribution, could be understood in the general context of the reduction of Courant algebroids. Below is an outline of those remarks. We plan to explore this in more detail in a forthcoming paper dealing with a general configuration space $Q$, rather than the special case of $Q=G$ of the present paper. 
Let $P$ be a manifold which is a $G$-principal bundle over $B=P / G$. One can consider the bundle $E=\left(T P \oplus T^{*} P\right) / G$ over $P / G=B$. It is easily checked that the natural lift of the $G$-action to $T P \oplus T^{*} P$ preserves the natural paring (denoted $\langle\langle\cdot, \cdot\rangle\rangle$ in Section 2), as well as the Courant bracket, which were introduced in Courant [23]. Thus, $E$ is naturally a Courant algebroid over $B$ in the general sense of Liu, Weinstein and $\mathrm{Xu}$ [36]. While this Courant algebroid is not of the form $T B \oplus T^{*} B$, one can still talk about Dirac structures in $E$. In fact, it is a general fact that if $D$ is a $G$-invariant Dirac structure in $T P \oplus T^{*} P$, then $D / G$ is a Dirac subbundle of $E$ (and $D / G$ is integrable if $D$ is, since $G$ preserves the Courant bracket). In the above general context, the reviewer suggested that an alternative proof of Theorem 4.2 (as well as its generalization, Theorem 7.2-which is the case with a distribution that will be introduced in Section 7), can be given.

In fact, in the context of Theorem 4.2, with $P=T^{*} G$ and using the isomorphism $T^{*} G=G \times \mathfrak{g}^{*}$, one gets a Courant algebroid $E=\mathfrak{g}^{*} \times\left(V \oplus V^{*}\right)$ viewed as a trivial bundle over $\mathfrak{g}^{*}$. Let $D$ be the canonical Dirac structure on $P=T^{*} G$ given by Eq. (4.1). Then, the quotient $D / G$, viewed as a structure on the bundle $E$ over $B=\mathfrak{g}^{*}$, gives $\mu$-dependent Dirac structures in $V \oplus V^{*}$, which is just a fiber of $E$ over $\mu \in \mathfrak{g}^{*}$. For the case of Dirac reduction with constraints in which a Dirac structure on $P=T^{*} G$ induced from a nontrivial distribution on $G$ is given, we will develop Theorem 7.2 later in Section 7, which could be also understood in the above context of reduction of Courant algebroids.

Note that the reduced Dirac structure in Theorem 4.2 is integrable with respect to the natural Courant bracket in $E$, whereas the one in Theorem 7.2 of course might not be. Some relevant works that deal with the reduction of Courant algebroids and associated Dirac structures can be found in, for instance, [12, 13], where the authors seem to focus mainly on so-called "exact" Courant algebroids (in the set up above, their reduction would yield $T B \oplus T^{*} B$, rather than $\left.E / G\right)$.

In this paper, our main goal here is to show how Lie-Dirac reduction can naturally provide the reduction procedures for the standard implicit Lagrangian and Hamiltonian systems, which can directly give us the implicit analogue of the Euler-Poincaré equations as well as the Lie-Poisson equations, together with their generalization to the case with a nontrivial distribution, and with applications to the Suslov problem in nonholonomic mechanics. In addition, we have made links with variational structures through the use of the Hamilton-Pontryagin principle; it is not clear to us if or how these principles fit into the context of Courant algebroids. We will not further explore the context of Courant algebroids in this paper. It is of course an interesting topic for future work, especially, for the case, which is planned for a subsequent paper, of "Dirac bundle reduction" in which one has a principal bundle $\pi: Q \rightarrow Q / G$ with a Lie group $G$ acting freely and properly on $Q$ and where there can be a nontrivial distribution on $Q$. The goal in that case would be to understand the general Dirac reduction of implicit Lagrangian and Hamiltonian systems as well as their relation to a reduced Hamilton-Pontryagin principle. 


\section{Euler-Poincaré-Dirac reduction}

This section develops the reduction of implicit Lagrangian systems in the context of the reduced Dirac structure $\mathcal{D}_{\mu}^{/ G}$ on $V=\mathfrak{g} \oplus \mathfrak{g}^{*}$. In particular, it is shown that the implicit Euler-Poincaré equations can be obtained by reducing the standard implicit Lagrangian system on the Pontryagin bundle $T G \oplus T^{*} G$.

\section{Standard implicit Lagrangian systems on $T G \oplus T^{*} G$}

Let $X: T G \oplus T^{*} G \rightarrow T T^{*} G$ be a partial vector field on $T^{*} G, L$ be a left invariant Lagrangian (possibly degenerate) on $T G$, and $D$ be the canonical Dirac structure given in Eq. (4.1). Using local coordinates $(g, v, p)$ for $T G \oplus T^{*} G, X$ will be written, at a point $(g, v, p) \in T G \oplus T^{*} G$ as

$$
X(g, v, p)=(g, p, \dot{g}, \dot{p}),
$$

where, as in the general theory, $\dot{g}, \dot{p}$ are functions of $(g, v, p)$. From (2.2), the Dirac differential of $L$, namely, $\mathfrak{D} L$ is locally given by

$$
\mathfrak{D} L=\left(g, \frac{\partial L}{\partial v},-\frac{\partial L}{\partial g}, v\right) .
$$

A standard implicit Lagrangian system $(L, T G, X)$ satisfies the condition

$$
(X, \mathfrak{D} L) \in D,
$$

which is locally denoted by, for each $(g, v) \in T G$,

$$
(X(g, v, p), \mathfrak{D} L(g, v)) \in D(g, p) .
$$

Equality of base points gives

$$
p=\frac{\partial L}{\partial v},
$$

and then the condition $(X, \mathfrak{D} L) \in D$ gives

$$
\left\langle-\frac{\partial L}{\partial g}, u\right\rangle+\langle\alpha, v\rangle=\langle\alpha, \dot{g}\rangle-\langle\dot{p}, u\rangle
$$

for all $u$ and all $\alpha$. Thus, we obtain

$$
p=\frac{\partial L}{\partial v}, \quad \dot{g}=v, \quad \dot{p}=\frac{\partial L}{\partial g},
$$

which are equivalent with the implicit Euler-Lagrange equations on $T G \oplus T^{*} G$ in Eq. (3.2).

\section{Reduction of the Dirac differential of Lagrangians}

Since the Lagrangian $L: T G \rightarrow \mathbb{R}$ is left invariant on $T G$, we have

$$
L\left(T_{g} L_{h} \cdot v\right)=L(v)
$$


for all $g, h \in G$ and $v \in T_{g} G$.

Recall that the differential of $L$ is the map $\mathbf{d} L: T G \rightarrow T^{*} T G$, whose coordinate expression is given, using local coordinates $(g, v) \in T G$, by

$$
\mathbf{d} L=\left(g, v, \frac{\partial L}{\partial g}, \frac{\partial L}{\partial v}\right) .
$$

Recall that there exists the natural diffeomorphism $\gamma_{G}: T^{*} T G \rightarrow T^{*} T^{*} G$ and that the Dirac differential of $L$ is the map $\mathfrak{D} L: T G \rightarrow T^{*} T^{*} G$ that is represented in coordinates by

$$
\mathfrak{D} L=\left(g, \frac{\partial L}{\partial v},-\frac{\partial L}{\partial g}, v\right) .
$$

The naive quotient of $\mathbf{d} L: T G \rightarrow T^{*} T G$ by $G$ is the map

$$
\mathbf{d}^{/ G} L: T G / G \rightarrow\left(T^{*} T G\right) / G,
$$

where $\mathbf{d}^{/ G}$ denotes the quotient of the differential operator by $G$. Since the diffeomorphism $\gamma_{G}: T^{*} T G \rightarrow T^{*} T^{*} G$ is $G$-equivariant, we can define a quotient map

$$
\gamma_{G}^{/ G}:\left(T^{*} T G\right) / G \rightarrow\left(T^{*} T^{*} G\right) / G
$$

and therefore, we may define the quotient of the Dirac differential of $L$ as

$$
\mathfrak{D}^{/ G} L=\gamma_{G}^{/ G} \circ \mathbf{d}^{/ G} L: T G / G \rightarrow\left(T^{*} T^{*} G\right) / G .
$$

\section{Left trivialized expressions}

Utilizing the left trivializing diffeomorphism

$$
\lambda: T G \rightarrow G \times \mathfrak{g}: v_{g} \mapsto\left(g, \eta=T_{g} L_{g^{-1}} v\right),
$$

one has the left invariant Lagrangian $\bar{L}=L \circ \lambda^{-1}$ induced on $G \times \mathfrak{g}$ and its differential may be represented by the map

$$
\mathbf{d} \bar{L}: G \times \mathfrak{g} \rightarrow(G \times \mathfrak{g}) \times\left(\mathfrak{g}^{*} \oplus \mathfrak{g}^{*}\right),
$$

which is expressed in coordinates by

$$
\mathbf{d} \bar{L}=\left(g, \eta, T_{e}^{*} L_{g} \frac{\partial \bar{L}}{\partial g}, \frac{\partial \bar{L}}{\partial \eta}\right) .
$$

Associated with $\gamma_{G}: T^{*} T G \rightarrow T^{*} T^{*} G$, we can naturally define the trivializing diffeomorphism

$$
\bar{\gamma}_{G}:(G \times \mathfrak{g}) \times\left(\mathfrak{g}^{*} \oplus \mathfrak{g}^{*}\right) \rightarrow\left(G \times \mathfrak{g}^{*}\right) \times\left(\mathfrak{g}^{*} \oplus \mathfrak{g}\right),
$$

which is given in coordinates as

$$
(g, \eta, v, \mu) \mapsto(g, \mu,-v, \eta) .
$$


Then, the Dirac differential of $\bar{L}$ becomes

$$
\mathfrak{D} \bar{L}=\bar{\gamma}_{G} \circ \mathbf{d} \bar{L},
$$

which is locally given by

$$
\mathfrak{D} \bar{L}=\left(g, \frac{\partial \bar{L}}{\partial \eta},-T_{e}^{*} L_{g} \frac{\partial \bar{L}}{\partial g}, \eta\right) .
$$

Definition 5.1. Since the Lagrangian $\bar{L}$ is $G$-invariant, we may write it as

$$
\bar{L}(g, \eta)=l(\eta)
$$

and define the quotient of the map $\mathbf{d} \bar{L}: G \times \mathfrak{g} \rightarrow(G \times \mathfrak{g}) \times\left(\mathfrak{g}^{*} \oplus \mathfrak{g}^{*}\right)$ by the map

$$
\mathbf{d}^{/ G} l: \mathfrak{g} \rightarrow \mathfrak{g} \times\left(\mathfrak{g}^{*} \oplus \mathfrak{g}^{*}\right),
$$

which is locally given by

$$
\mathbf{d}^{/ G} l=\left(\eta, 0, \frac{\delta l}{\delta \eta}\right) .
$$

Further, the trivializing diffeomorphism $\bar{\gamma}_{G}$ is $G$-equivariant, and so we can define the quotient map

$$
\bar{\gamma}_{G}^{/ G}: \mathfrak{g} \times\left(\mathfrak{g}^{*} \oplus \mathfrak{g}^{*}\right) \rightarrow \mathfrak{g}^{*} \times\left(\mathfrak{g}^{*} \oplus \mathfrak{g}\right),
$$

which is given in coordinates as

$$
(\eta, v, \mu) \mapsto(\mu,-v, \eta) .
$$

The reduced Dirac differential for $l$ is defined to be

$$
\mathfrak{D}^{/ G} l=\bar{\gamma}_{G}^{/ G} \circ \mathbf{d}^{/ G} l: \mathfrak{g} \rightarrow \mathfrak{g}^{*} \times\left(\mathfrak{g}^{*} \oplus \mathfrak{g}\right),
$$

which is locally denoted by

$$
\mathfrak{D}^{/ G} l=\left(\frac{\delta l}{\delta \eta}, 0, \eta\right),
$$

where $\mathfrak{D}^{/ G}$ is the reduced Dirac differential operator for $l$.

REMARK. The differential of the reduced Lagrangian $l: \mathfrak{g} \rightarrow \mathbb{R}$ is naturally given by the map

$$
\mathbf{d} l: \mathfrak{g} \rightarrow \mathfrak{g} \times \mathfrak{g}^{*},
$$

which is locally expressed at a point $\eta \in \mathfrak{g}$ as

$$
\mathbf{d} l=\left(\eta, \frac{\delta l}{\delta \eta}\right) .
$$

Note that the map $\mathbf{d} l: \mathfrak{g} \rightarrow \mathfrak{g} \times \mathfrak{g}^{*}$ is not the same as the map $\mathbf{d}^{/ G} l: \mathfrak{g} \rightarrow \mathfrak{g} \times\left(\mathfrak{g}^{*} \oplus \mathfrak{g}^{*}\right)$. 


\section{Reduction of partial vector fields}

Let $X: T G \oplus T^{*} G \rightarrow T T^{*} G$ be a left invariant partial vector field on $T^{*} G$, which is locally written, at a point $(g, v, p) \in T G \oplus T^{*} G$, as

$$
X(g, v, p)=(g, p, \dot{g}, \dot{p}) .
$$

Since $X$ is left invariant, one has

$$
\left(T_{g} L_{h}\right) X(g, v, p)=X\left(h g, T_{g} L_{h} \cdot v, T_{h g}^{*} L_{h^{-1}} \cdot p\right) .
$$

By using the trivializing diffeomorphisms $\lambda: T G \rightarrow G \times \mathfrak{g}$ and $\bar{\lambda}: T^{*} G \rightarrow G \times \mathfrak{g}^{*}$, which can be thought of together as a map $\Lambda: T G \oplus T^{*} G \rightarrow G \times\left(\mathfrak{g} \oplus \mathfrak{g}^{*}\right)$, let $\mathcal{X}: G \times\left(\mathfrak{g} \oplus \mathfrak{g}^{*}\right) \rightarrow T\left(G \times \mathfrak{g}^{*}\right) \cong\left(G \times \mathfrak{g}^{*}\right) \times\left(\mathfrak{g} \oplus \mathfrak{g}^{*}\right)$ be the trivialized expression of $X$ given by, for each $(g, \eta, \mu) \in G \times\left(\mathfrak{g} \oplus \mathfrak{g}^{*}\right)$,

$$
\mathcal{X}(g, \eta, \mu)=(g, \mu, \xi, \dot{\mu}) \in\left(G \times \mathfrak{g}^{*}\right) \times\left(\mathfrak{g} \oplus \mathfrak{g}^{*}\right),
$$

where $\eta=T_{g} L_{g^{-1}} v, \xi=T_{g} L_{g^{-1}} \dot{g}$ and $\mu=T_{e}^{*} L_{g} \cdot p$ such that $\mu=\mathbb{F} l(\eta)$. The map $\mathcal{X}$ is left invariant since $X$ is left invariant and $\lambda, \bar{\lambda}$ and $\Lambda$ are equivariant. That is,

$$
\left(T_{g} L_{h}\right) \mathcal{X}(g, \eta, \mu)=\mathcal{X}(h g, \eta, \mu),
$$

where

$$
\mathcal{X}(h g, \eta, \mu)=(h g, \mu, \xi, \dot{\mu}) \in\left(G \times \mathfrak{g}^{*}\right) \times\left(\mathfrak{g} \oplus \mathfrak{g}^{*}\right) .
$$

DEFINITION 5.2. The reduction of the partial vector field $\mathcal{X}: G \times\left(\mathfrak{g} \oplus \mathfrak{g}^{*}\right) \rightarrow$ $\left(G \times \mathfrak{g}^{*}\right) \times\left(\mathfrak{g} \oplus \mathfrak{g}^{*}\right)$ is defined by the quotient of $\mathcal{X}$ by $G$ as

$$
\mathcal{X}^{/ G}: \mathfrak{g} \oplus \mathfrak{g}^{*} \rightarrow \mathfrak{g}^{*} \times\left(\mathfrak{g} \oplus \mathfrak{g}^{*}\right),
$$

which will be written as

$$
\mathcal{X}^{/ G}(\eta, \mu)=(\mu, \xi, \dot{\mu}) \in \mathfrak{g}^{*} \times\left(\mathfrak{g} \oplus \mathfrak{g}^{*}\right),
$$

where $\xi$ and $\dot{\mu}$ are functions of $(\eta, \mu)$.

\section{Euler-Poincaré-Dirac reduction}

Now we have the needed basic ingredients to define what we shall refer to as the Euler-Poincaré-Dirac reduction procedure. This will make use of the $\mu$-dependent reduced Dirac structure $\mathcal{D}_{\mu}^{/ G}$ on $V=\mathfrak{g} \oplus \mathfrak{g}^{*}$; together with the reduced Lagrangian, this will induce the implicit Euler-Poincaré equations.

Definition 5.3. Let $(L, T G, X)$ be a standard implicit Lagrangian system and let, for fixed $\mu \in \mathfrak{g}^{*}, \mathcal{D}_{\mu}^{/ G}$ be the reduced Dirac structure on $V=\mathfrak{g} \oplus \mathfrak{g}^{*}$, which is given by Eq. (4.6), or equivalently, (4.8).

The reduction of a standard implicit Lagrangian system $(L, T G, X)$ is a triple $\left(l, \mathfrak{g}, \mathcal{X}^{/ G}\right)$, where $l=L \mid \mathfrak{g}: \mathfrak{g} \rightarrow \mathbb{R}$ is the reduced Lagrangian and a reduced partial 
vector field $\mathcal{X}^{/ G}: \mathfrak{g} \oplus \mathfrak{g}^{*} \rightarrow \mathfrak{g}^{*} \times\left(\mathfrak{g} \oplus \mathfrak{g}^{*}\right)$ that satisfies, for each $\eta \in \mathfrak{g}$, the condition

$$
\left(\mathcal{X}^{/ G}(\eta, \mu), \mathfrak{D}^{/ G} l(\eta)\right) \in \mathcal{D}_{\mu}^{/ G} \text {. }
$$

Notice that for this to make sense, the base points must all coincide with $\mu$; that is, the condition $\mu=\mathbb{F} l(\eta) \in \mathfrak{g}^{*}$ must hold.

DEFINITION 5.4. A solution curve of the reduced standard implicit Lagrangian systems $\left(l, \mathfrak{g}, \mathcal{X}^{/ G}\right)$ is a curve $(\eta(t), \mu(t))$ in $\mathfrak{g} \oplus \mathfrak{g}^{*}$ such that the time derivative of $\mu(t)$ is equal to $\dot{\mu}$ and we also have base point equality, namely, $\mu(t)=\mathbb{F} l(\eta(t)) \in \mathfrak{g}^{*}$.

Proposition 5.5. Let $\left(l, \mathfrak{g}, \mathcal{X}^{/ G}\right)$ be the reduction of a standard implicit Lagrangian system on $T G \oplus T^{*} G$; that is, it satisfies Eq. (5.3). Let $(\eta(t), \mu(t))$ be an integral curve of $\mathcal{X}^{/ G}$. Then the implicit Euler-Poincaré equations hold, namely,

$$
\mu=\frac{\delta l}{\delta \eta}, \quad \xi=\eta, \quad \dot{\mu}=\operatorname{ad}_{\xi}^{*} \mu .
$$

Proof: Substituting Eqs. (5.1) and (5.2) into the condition of Eq. (5.3), namely,

$$
\left(\mathcal{X}^{/ G}(\eta, \mu), \mathfrak{D}^{/ G} l(\eta)\right) \in \mathcal{D}_{\mu}^{/ G}
$$

for each $\eta \in \mathfrak{g}$ and with $\mu=\delta l / \delta \eta \in \mathfrak{g}^{*}$, it follows, at fixed $\mu \in \mathfrak{g}^{*}$,

$$
((\xi, \dot{\mu}),(0, \eta)) \in \mathcal{D}_{\mu}^{/ G}
$$

where $\mathcal{X}^{/ G}(\eta, \mu)=(\mu, \xi, \dot{\mu})$ and $\mathfrak{D}^{/ G} l(\eta)=(\delta l / \delta \eta, 0, \eta)$ together with $\mu=\delta l / \delta \eta$. In view of Eq. (4.8), it immediately yields Eq. (5.4).

Thus, we can formulate implicit Euler-Poincaré equations on $V=\mathfrak{g} \oplus \mathfrak{g}^{*}$ in the context of the reduced Dirac structure $\mathcal{D}_{\mu}^{/ G}$, which are quite equivalent with the ones derived from the reduced Hamilton-Pontryagin principle in Eq. (3.3).

\section{Implicit Euler-Poincaré reconstruction of dynamics}

As shown in Eq. (5.4), implicit Euler-Poincaré equations consist of equations of motion $\dot{\mu}=\operatorname{ad}_{\xi}^{*} \mu$, the partial Legendre transformation $\mu=\delta l / \delta \eta$ together with the kinematic equation $\xi=\eta$. Noting that $\xi(t)=T_{g} L_{g^{-1}} \dot{g}(t) \in \mathfrak{g}$ and $\eta(t)=T_{g} L_{g}-1 v(t) \in \mathfrak{g}$, the kinematic equation $\xi(t)=\eta(t)$ is the reduction of the second-order condition $\dot{g}(t)=v(t)$ in the implicit Euler-Lagrange equations in Eq. (3.2). This is the context of implicit Euler-Poincaré reconstruction of dynamics given by the following theorem.

THEOREM 5.6. Let $G$ be a Lie group, let $L$ be a left invariant Lagrangian (possibly degenerate) on $T G$ and $l=L \mid \mathfrak{g}$ be the reduced Lagrangian. Let $g_{0} \in G$, $v_{0} \in T_{g_{0}} G$ and $\eta_{0}=T_{g_{0}} L_{g_{0}^{-1}} v_{0} \in \mathfrak{g}$. Let $(\eta(t), \mu(t)) \in \mathfrak{g} \oplus \mathfrak{g}^{*}$ be a solution curve of the implicit Euler-Poincaré equations with initial conditions $\eta(0)=\eta_{0}$ and let $p_{0} \in T_{g_{0}}^{*} G$ be such that $\mu(0)=T_{e}^{*} L_{g_{0}}\left(p_{0}\right)$; that is, assume that the equations

$$
\dot{\mu}(t)=\operatorname{ad}_{\xi(t)}^{*} \mu(t),
$$


and the reduced Legendre transformation

$$
\mu(t)=\left(\frac{\delta l}{\delta \eta}\right)(t),
$$

together with the kinematic equations $\xi(t)=\eta(t)$ hold.

Then, the solution curve $(g(t), v(t), p(t)) \in T G \oplus T^{*} G$ of the implicit EulerLagrange equations

$$
\dot{g}=v, \quad p=\frac{\partial L}{\partial v}, \quad \dot{p}=\frac{\partial L}{\partial g}
$$

with initial condition $\left(g_{0}, v_{0}, p_{0}\right)$ is given as follows; let $g(t)$ be the solution of the equation $T_{g} L_{g^{-1}} \dot{g}=\xi$, namely,

$$
\frac{d g(t)}{d t}=T_{e} L_{g(t)} \xi(t)
$$

with initial condition $g(0)=g_{0}$. Then, $v(t)=g(t) \eta(t)$ and $p(t)=g(t) \mu(t)$; that is,

$$
v(t)=T_{e} L_{g(t)} \eta(t) \quad \text { and } \quad p(t)=T_{g(t)}^{*} L_{(g(t))^{-1}} \mu(t) .
$$

Proof: By construction, the curve $v(t)$ satisfies the given initial conditions, and the condition $\dot{g}=v$. Also, by construction, $p(t)$ is given by the Legendre transformation and satisfies the given initial conditions. To check that it satisfies the equations $\dot{p}=\partial L / \partial g$, note that by construction,

$$
\bar{\lambda}(p(t))=\left(g(t), T_{e}^{*} L_{g(t)} p(t)\right)=(g(t), \mu(t))
$$

and that the evolution equation for $\mu(t)$ is, by construction, the reduction of the equation for $p(t)$.

\section{Lie-Poisson-Dirac reduction}

If a given Lagrangian is regular, we can define a regular Hamiltonian on $T^{*} G$ via the Legendre transform. In this section, we make a Hamiltonian analogue of the Euler-Poincaré-Dirac reduction, namely, a reduction procedure called Lie-PoissonDirac reduction for the standard implicit Hamiltonian system on $T^{*} G$, which yields implicit Lie-Poisson equations on $\mathfrak{g} \oplus \mathfrak{g}^{*}$.

\section{Implicit Hamiltonian systems}

Before going into details, let us briefly review implicit Hamiltonian systems associated with an induced Dirac structure along Yoshimura and Marsden [51].

Let $Q$ be a configuration manifold. Given a Lagrangian $L$ on $T Q$ and the Legendre transform $\mathbb{F} L: T Q \rightarrow T^{*} Q$ is locally given by, for each $(q, v) \in T Q$,

$$
\mathbb{F} L(q, v)=\left(q, \frac{\partial L}{\partial v}\right) .
$$


When $L$ is regular, we can define a regular Hamiltonian $H$ on $T^{*} Q$ by using the Legendre transformation $\mathbb{F} L: T Q \rightarrow T^{*} Q$ such that

$$
H=E \circ \mathbb{F} L^{-1},
$$

where $E(q, v)=\left\langle\mathbb{F} L(q, v), v_{q}\right\rangle-L(q, v)$ is an energy on $T Q$.

The definition of an implicit Hamiltonian system associated with an induced Dirac structure on $T^{*} Q$ is given as follows: Let $\Delta_{Q}$ be a distribution on $Q$ and $X$ be a vector field on $T^{*} Q$. Let $D_{\Delta_{Q}} \subset T T^{*} Q \oplus T^{*} T^{*} Q$ be an induced Dirac structure on $T^{*} Q$. Then, an implicit Hamiltonian system is a triple $\left(H, \Delta_{Q}, X\right)$, which satisfies, for each $(q, p) \in T^{*} Q$,

$$
(X(q, p), \mathbf{d} H(q, p)) \in D_{\Delta_{Q}}(q, p) .
$$

\section{Standard implicit Hamiltonian systems on $T^{*} G$}

Let us consider a standard implicit Hamiltonian system on $T^{*} G$; the case in which $\Delta_{Q}=T Q$ and $Q=G$, namely, a configuration space is given by a Lie group $G$ and $\Delta_{G}=T G$ there exits no kinematic constraints. Let $L$ be a regular left invariant Lagrangian on $T G$. Define a Hamiltonian $H$ on $T^{*} G$ by $H=E \circ(\mathbb{F} L)^{-1}$, where $\mathbb{F} L: T G \rightarrow T^{*} G$ and $E(g, v)=\left\langle\mathbb{F} L(g, v), v_{g}\right\rangle-L(g, v)$. Since $L$ being left invariant on $T G$ and $\mathbb{F} L: T G \rightarrow T^{*} G$ is diffeomorphism, $H$ is also to be left invariant on $T^{*} G$.

Recall from Eq. (4.1) that the canonical Dirac structure $D$ on $T^{*} G$ is given by, for each $p_{g} \in T^{*} G$,

$$
\begin{aligned}
D\left(p_{g}\right)=\left\{\left(v_{p_{g}}, \alpha_{p_{g}}\right) \in T_{p_{g}} T^{*} G \times T_{p_{g}}^{*} T^{*} G \mid \alpha_{p_{g}}\left(w_{p_{g}}\right)=\right. & \Omega\left(p_{g}\right)\left(v_{p_{g}}, w_{p_{g}}\right) \\
& \text { for all } \left.w_{p_{g}} \in T_{p_{g}} T^{*} G\right\} .
\end{aligned}
$$

Recall also that the canonical symplectic form $\Omega$ on $T^{*} G$ is represented, using local coordinates $(g, p)$ for $T^{*} G$, by

$$
\Omega\left(\left(g, p, u_{1}, \alpha_{1}\right),\left(g, p, u_{2}, \alpha_{2}\right)\right)=\left\langle\alpha_{2}, u_{1}\right\rangle-\left\langle\alpha_{1}, u_{2}\right\rangle .
$$

Let $X$ be a left invariant vector field on $T^{*} G$, which is denoted, in coordinates, by

$$
X=(g, p, \dot{g}, \dot{p})
$$

and the differential of the Hamiltonian may be written in coordinates as

$$
\mathbf{d} H=\left(g, p, \frac{\partial H}{\partial g}, \frac{\partial H}{\partial p}\right) .
$$

Then, the condition for the standard implicit Hamiltonian system $\left(H, \Delta_{G}=T G, X\right)$ is given by, for each $(g, p)$,

$$
(X(g, p), \mathbf{d} H(g, p)) \in D(g, p)
$$

and hence we obtain

$$
\left\langle\frac{\partial H}{\partial g}, u\right\rangle+\left\langle\alpha, \frac{\partial H}{\partial p}\right\rangle=\langle\alpha, \dot{g}\rangle-\langle\dot{p}, u\rangle
$$


for all $u$ and all $\alpha$. Thus, it follows

$$
\dot{g}=\frac{\partial H}{\partial p}, \quad \dot{p}=-\frac{\partial H}{\partial g},
$$

which implies that $(H, T G, X)$ naturally induces Hamilton's equations.

\section{Reduction of the differential operator for Hamiltonians}

Since $H$ is left invariant, we have

$$
H\left(T_{h g}^{*} L_{h^{-1}} \cdot p\right)=H(p)
$$

for all $g, h \in G$ and $p \in T_{g}^{*} G$. Hence, we can define the reduced Hamiltonian by

$$
H^{/ G}: T^{*} G / G \rightarrow \mathbb{R} \text {. }
$$

The differential of $H$ is the one-form on $T^{*} G$ that is the map

$$
\text { d } H: T^{*} G \rightarrow T^{*} T^{*} G,
$$

which is denoted, in local coordinates $(g, p)$ for $T^{*} G$, as

$$
\mathbf{d} H=\left(g, p, \frac{\partial H}{\partial g}, \frac{\partial H}{\partial p}\right) .
$$

Then, the naive quotient of $\mathbf{d} H: T^{*} G \rightarrow T^{*} T^{*} G$ may be defined as

$$
\mathbf{d}^{/ G} H:\left(T^{*} G\right) / G \rightarrow\left(T^{*} T^{*} G\right) / G .
$$

\section{Left trivialized expressions}

Employing the trivializing diffeomorphism

$$
\bar{\lambda}: T^{*} G \rightarrow G \times \mathfrak{g}^{*} ; \quad p_{g} \mapsto\left(g, \mu=T_{e}^{*} L_{g}\left(p_{g}\right)\right),
$$

the differential of the left invariant Hamiltonian $\bar{H}=H \circ \bar{\lambda}^{-1}$ induced on $G \times \mathfrak{g}^{*}$ is the map

$$
\mathbf{d} \bar{H}: G \times \mathfrak{g}^{*} \rightarrow\left(G \times \mathfrak{g}^{*}\right) \times\left(\mathfrak{g}^{*} \oplus \mathfrak{g}\right),
$$

which is expressed, using coordinates $(g, \mu) \in G \times \mathfrak{g}^{*}$, by

$$
\mathbf{d} \bar{H}=\left(g, \mu, T_{e}^{*} L_{g} \frac{\partial \bar{H}}{\partial g}, \frac{\partial \bar{H}}{\partial \mu}\right) .
$$

Definition 6.1. The induced Hamiltonian $\bar{H}$ on $G \times \mathfrak{g}^{*}$ is $G$-invariant, and it reads

$$
\bar{H}(g, \mu)=h(\mu),
$$

where $h: \mathfrak{g}^{*} \rightarrow \mathbb{R}$ is the reduced Hamiltonian. Define the reduction of the differential operator for $h$ by the map

$$
\mathbf{d}^{/ G} h: \mathfrak{g}^{*} \rightarrow \mathfrak{g}^{*} \times\left(\mathfrak{g}^{*} \oplus \mathfrak{g}\right),
$$


which is locally given by

$$
\mathbf{d}^{\prime G} h=\left(\mu, 0, \frac{\delta h}{\delta \mu}\right) .
$$

REMARKs. The differential of the reduced Hamiltonian $h: \mathfrak{g}^{*} \rightarrow \mathbb{R}$ is given by

$$
\mathbf{d} h: \mathfrak{g}^{*} \rightarrow \mathfrak{g}^{*} \times \mathfrak{g},
$$

which is locally expressed by, for $\mu \in \mathfrak{g}^{*}$,

$$
\mathbf{d} h=\left(\mu, \frac{\delta h}{\delta \mu}\right) .
$$

So, needless to say, $\mathbf{d} h$ is not equivalent with the map

$$
\mathbf{d}^{/ G} h: \mathfrak{g}^{*} \rightarrow \mathfrak{g}^{*} \times\left(\mathfrak{g}^{*} \oplus \mathfrak{g}\right),
$$

which we will utilize to reduce the standard implicit Hamiltonian systems.

Definition 6.2. Since $X$ is left invariant and by equivariance of $\bar{\lambda}: T^{*} G \rightarrow$ $G \times \mathfrak{g}^{*}$, we can define the induced vector field on $G \times \mathfrak{g}^{*}$ by $\mathcal{X}=\bar{\lambda}_{*} X$, which is written, at each point $(g, \mu) \in G \times \mathfrak{g}^{*}$, by

$$
\mathcal{X}(g, \mu)=(g, \mu, \dot{g}, \dot{\mu}),
$$

where $(g, \dot{g})$ is a partial vector field on $G$ smoothly depending on $\mu \in \mathfrak{g}^{*}$ and hence we write

$$
Y_{\mu}=(g, \dot{g}) \text {. }
$$

Since the vector field $Y_{\mu}$ is left invariant, we have, for all $f, g \in G$,

$$
T_{f} L_{g} Y_{\mu}(f)=Y_{\mu}(g f),
$$

which reads

$$
Y_{\mu}(g)=T_{e} L_{g} Y_{\mu}(e)
$$

Then, we can define a partial vector field

$$
\mathcal{X}^{/ G}: \mathfrak{g}^{*} \rightarrow \mathfrak{g}^{*} \times\left(\mathfrak{g} \oplus \mathfrak{g}^{*}\right)
$$

by the quotient of the map $\mathcal{X}: G \times \mathfrak{g}^{*} \rightarrow \mathfrak{g}^{*} \times\left(\mathfrak{g} \oplus \mathfrak{g}^{*}\right)$ by $G$, which is given, in coordinates, by

$$
\mathcal{X}^{/ G}=(\mu, \xi(\mu), \dot{\mu}),
$$

where $\xi(\mu)=T_{g} L_{g^{-1}} \dot{g}$.

\section{Lie-Poisson-Dirac reduction}

Let us define reduction of the standard implicit Hamiltonian system in the context of the reduced Dirac structure $\mathcal{D}_{\mu}^{\prime G}$ on $V=\mathfrak{g} \oplus \mathfrak{g}^{*}$.

Definition 6.3. Let $(H, T G, X)$ be the standard implicit Hamiltonian system associated with the canonical Dirac structure $D$ on $T^{*} G$. Let $\mathcal{D}=\bar{\lambda}_{*} D$ be the 
canonical Dirac structure on $G \times \mathfrak{g}^{*}$ given in Eq. (4.3). Let $\mathcal{X}^{/ G}$ be the partial vector field defined in Eq. (6.3) and $\mathcal{D}_{\mu}^{/ G}$ be the reduced Dirac structure on $V=\mathfrak{g} \oplus \mathfrak{g}^{*}$ fixed at $\mu \in \mathfrak{g}^{*}$, given in Eq. (4.6) or (4.8).

Then, the reduction of a standard implicit Hamiltonian system $(H, T G, X)$ may be given by a triple $\left(h, \mathfrak{g}, \mathcal{X}^{/ G}\right)$ that satisfies, at fixed $\mu \in \mathfrak{g}^{*}$,

$$
\left(\mathcal{X}^{/ G}(\mu), \mathbf{d}^{/ G} h(\mu)\right) \in \mathcal{D}_{\mu}^{/ G} \text {. }
$$

DEFINITION 6.4. A solution curve of the reduced standard implicit Hamiltonian system $\left(h, \mathfrak{g}, \mathcal{X}^{/ G}\right)$ is a curve $\mu(t)$ in $\mathfrak{g}^{*}$, which is an integral curve of the partial vector field $\mathcal{X}^{/ G}=(\mu, \xi(\mu), \dot{\mu})$ such that $\xi(t)=\mathbb{F} h(\mu(t))$.

PROPOSITION 6.5. The reduction of the standard implicit Hamiltonian system given in Eq. (6.4) induces implicit Lie-Poisson equations on $\mathfrak{g} \oplus \mathfrak{g}^{*}$ as

$$
\xi=\frac{\delta h}{\delta \mu}, \quad \dot{\mu}=\operatorname{ad}_{\xi}^{*} \mu .
$$

Proof: Substituting Eqs. (6.1) and (6.3) into the condition of Eq. (6.4), namely,

$$
\left(\mathcal{X}^{/ G}(\mu), \mathbf{d}^{/ G} h(\mu)\right) \in \mathcal{D}_{\mu}^{/ G},
$$

where $\mathcal{X}^{/ G}=(\mu, \xi(\mu), \dot{\mu})$ and $\mathbf{d}^{/ G} h=(\mu, 0, \delta h / \delta \mu)$. In view of Eq. (4.8), we obtain Eq. (6.5).

Thus, we obtain implicit Lie-Poisson equations on $\mathfrak{g} \oplus \mathfrak{g}^{*}$ in the context of the reduced Dirac structure, which are quite equivalent with the ones previously derived from the Lie-Poisson variational principle in Eq. (3.8).

\section{Reconstruction of dynamics}

The implicit Lie-Poisson equations in Eq. (6.5) consist of equations of motion $\dot{\mu}=\operatorname{ad}_{\xi}^{*} \mu$ and the partial Legendre transformation $\xi=\delta h / \delta \mu$. Here, we shall show how the reconstruction of dynamics can be done from implicit Lie-Poisson equations. Implicit Lie-Poisson reconstruction of dynamics can be given by the following theorem.

THEOREM 6.6. Let $H$ be a left invariant Hamiltonian on $T^{*} G$ and $h$ be the reduced Hamiltonian on $\mathfrak{g}^{*}$. Let $\mu(t) \in \mathfrak{g}^{*}$ be a solution curve of the implicit Lie-Poisson equations

$$
\dot{\mu}(t)=\operatorname{ad}_{\xi(t)}^{*} \mu(t)
$$

with the initial condition $\mu(0)=T_{e}^{*} L_{g_{0}}\left(\alpha_{g_{0}}\right)$, which are accompanied with

$$
\xi(t)=\left(\frac{\delta h}{\delta \mu}\right)(t) .
$$


Then, the integral curve $p(t) \in T_{g(t)}^{*} G$ of a vector field $X$ on $T^{*} G$ with initial condition $p(0)=p_{g_{0}}$ is given by

$$
p(t)=T_{g(t)}^{*} L_{(g(t))^{-1}} \mu(t),
$$

where $g(t)$ is the solution of the equation $T_{g} L_{g-1} \dot{g}=\xi$, namely,

$$
\frac{d g(t)}{d t}=T_{e} L_{g(t)} \xi(t)
$$

with initial condition $g(0)=g_{0}$.

Proof: The curve $p(t)$ is the unique integral curve of $X$ with initial condition $p(0)=p_{g(0)}$ if and only if

$$
\bar{\lambda}(p(t))=\left(g(t), T_{e}^{*} L_{g(t)} p_{g(t)}(t)\right)=:(g(t), \mu(t))
$$

is the integral curve of the induced vector field $\mathcal{X}=\bar{\lambda}_{*} X$ on $G \times \mathfrak{g}^{*}$, which is given in Eq. (6.2), together with initial condition

$$
\bar{\lambda}(p(0))=\left(g_{0}, T_{e}^{*} L_{g_{0}} p_{0}\right) .
$$

\section{Dirac reduction with constraints}

In this section, we shall investigate the reduction of an induced Dirac structure on the cotangent bundle, for the case in which a configuration manifold is given by a Lie group and with a left invariant constraint distribution. It will be shown that this Dirac reduction plays a key role in the reduction of implicit Lagrangian and Hamiltonian systems on $T G \oplus T^{*} G$ for the so-called Suslov problems in nonholonomic mechanics.

\section{The induced Dirac structure on $T^{*} G$}

Let $\Delta_{G} \subset T G$ be a constraint distribution on $G$, where we assume that the distribution is regular. Assume also that the distribution $\Delta_{G}$ is left invariant under the group action $L_{h}: G \rightarrow G: g \mapsto h g$ such that the subspace $\Delta_{G}(g) \subset T_{g} G$ is mapped by the tangent of the group action to the subspace $\Delta_{G}(h g) \subset T_{h g} G$. Define the distribution on $T^{*} G$ by

$$
\Delta_{T * G}=\left(T \pi_{G}\right)^{-1}\left(\Delta_{G}\right),
$$

where $\pi_{G}: T^{*} G \rightarrow G$ is the cotangent projection. Recall that an induced Dirac structure $D_{\Delta_{G}}$ on $T^{*} G$ is defined, for each $p_{g} \in T^{*} G$, by

$$
\begin{aligned}
& D_{\Delta_{G}}\left(p_{g}\right)=\left\{\left(v_{p_{g}}, \alpha_{p_{g}}\right) \in T_{p_{g}} T^{*} G \times T_{p_{g}}^{*} T^{*} G \mid v_{p_{g}} \in \Delta_{T^{*} G}\left(p_{g}\right),\right. \text { and } \\
& \left.\alpha_{p_{g}}\left(w_{p_{g}}\right)=\Omega\left(p_{g}\right)\left(v_{p_{g}}, w_{p_{g}}\right) \quad \text { for all } \quad w_{p_{g}} \in \Delta_{T^{*} G}\left(p_{g}\right)\right\},
\end{aligned}
$$

where $\Omega$ is the canonical two-form on $T^{*} G$. 


\section{Trivialized expressions}

Let us choose local coordinates $g$ for $G$ so that $G$ is locally represented by an open set $U \subset \mathbb{R}^{n}$. The constraint set $\Delta_{G}$ defines a subspace of $T_{g} G$ at each point $g \in G$, which we locally denote by $\Delta(g) \subset \mathbb{R}^{n}$ at each point $g \in U$. If we let the dimension of the constraint space be $n-m$, then we can choose a basis $e_{m+1}(g), e_{m+2}(g), \ldots, e_{n}(g)$ of $\Delta(g)$. It is also common to represent constraint sets as the simultaneous kernel of a number of constraint one-forms, namely, the annihilator of $\Delta(g)$, denoted by $\Delta^{\circ}(g)$, is spanned by such one-forms, which we write as $\omega^{1}, \omega^{2}, \ldots, \omega^{m}$. Hence, the local expression of $\Delta_{G} \subset T G$ is given by, for each $g \in U$,

$$
\Delta_{G}(g)=\left\{v \in T_{g} G \mid v \in \Delta(g)\right\} .
$$

The distribution $\Delta_{G}$ is left invariant, and it reads

$$
\Delta_{G}(h g)=\Delta_{G}(g)
$$

for all $h, g \in G$, where

$$
\Delta_{G}(h g)=\left\{T_{g} L_{h} v \in T_{h g} G \mid T_{g} L_{h} v \in \Delta(h g)\right\} .
$$

We can define the distribution $\Delta_{G \times \mathfrak{g}^{*}}$ on $G \times \mathfrak{g}$ by

$$
\Delta_{G \times \mathfrak{g}^{*}}=\left(T \bar{\pi}_{G}\right)^{-1}\left(\Delta_{G}\right) \subset T\left(G \times \mathfrak{g}^{*}\right) .
$$

In the above, $\bar{\pi}_{G}: G \times \mathfrak{g}^{*} \rightarrow G$ is defined such that $\pi_{G}=\bar{\pi}_{G} \circ \bar{\lambda}$, where $\pi_{G}: T^{*} G \rightarrow G$ is the canonical projection and $\bar{\lambda}: T^{*} G \rightarrow G \times \mathfrak{g}^{*}$. Employing local coordinates $(g, \mu)$ for $G \times \mathfrak{g}^{*}$ and $(v, \rho)$ for $T_{(g, \mu)}\left(G \times \mathfrak{g}^{*}\right) \cong T_{g} G \times \mathfrak{g}^{*}$, the distribution $\Delta_{G \times \mathfrak{g}^{*}} \subset T\left(G \times \mathfrak{g}^{*}\right)$ can be locally represented by, for each $(g, \mu) \in G \times \mathfrak{g}^{*}$,

$$
\Delta_{G \times \mathfrak{g}^{*}}(g, \mu)=\left\{(v, \rho) \in T_{g} G \times \mathfrak{g}^{*} \mid v \in \Delta(g)\right\} .
$$

Since $\Delta_{G}$ is left-invariant, it is easily verified that the distribution $\Delta_{G \times \mathfrak{g}^{*}}$ is also left-invariant, namely,

$$
\Delta_{G \times \mathfrak{g}^{*}}(h g, \mu)=\Delta_{G \times \mathfrak{g}^{*}}(g, \mu)
$$

for all $h, g \in G$ and $\mu \in \mathfrak{g}^{*}$, where

$$
\Delta_{G \times \mathfrak{g}^{*}}(h g, \mu)=\left\{\left(T_{g} L_{h} v, \rho\right) \in T_{h g} G \times \mathfrak{g}^{*} \mid T_{g} L_{h} v \in \Delta(h g)\right\} .
$$

Now, we can define an induced Dirac structure on $G \times \mathfrak{g}^{*}$ by

$$
\mathcal{D}_{\Delta_{G}}=\bar{\lambda}_{*} D_{\Delta_{G}}
$$

which is locally given by, for each $(g, \mu) \in G \times \mathfrak{g}^{*}$,

$$
\begin{gathered}
\mathcal{D}_{\Delta_{G}}(g, \mu)=\left\{(v, \rho),(\beta, \eta) \in\left(T_{g} G \times \mathfrak{g}^{*}\right) \times\left(T_{g}^{*} G \times \mathfrak{g}^{*}\right) \mid(v, \rho) \in \Delta_{G \times \mathfrak{g}^{*}}(g, \mu),\right. \\
\text { and } \left.\langle\beta, w\rangle+\langle\sigma, \eta\rangle=\omega(g, \mu)((v, \rho),(w, \sigma)) \text { for all }(w, \sigma) \in \Delta_{G \times \mathfrak{g}^{*}}(g, \mu)\right\},
\end{gathered}
$$

where $\omega=\bar{\lambda}_{*} \Omega$ is the canonical symplectic structure on $G \times \mathfrak{g}^{*}$ given in Eq. (4.2). 


\section{Invariance of induced Dirac structures}

Let $\Lambda$ be a left $G$-action on $G \times \mathfrak{g}^{*}$ and hence $\Lambda_{h}(g, \mu)=(h g, \mu)$. Since the canonical symplectic structure $\omega$ and the distribution $\Delta_{G \times \mathfrak{g}^{*}}$ are $G$-invariant, an induced Dirac structure $\mathcal{D}_{\Delta_{G}}$ on $G \times \mathfrak{g}^{*}$ is $G$-invariant as

$$
\left(\Lambda_{h^{*}} X,\left(\Lambda_{h}^{*}\right)^{-1} \alpha\right) \in \mathcal{D}_{\Delta_{G}}
$$

for all $(X, \alpha) \in \mathcal{D}_{\Delta_{G}}$. Namely, the left-invariance of an induced Dirac structure $\mathcal{D}_{\Delta_{G}}$ is locally represented by

$$
\mathcal{D}_{\Delta_{G}}(h g, \mu)=\mathcal{D}_{\Delta_{G}}(g, \mu),
$$

for all $h, g \in G$ and $\mu \in \mathfrak{g}^{*}$, where

$$
\begin{aligned}
\mathcal{D}_{\Delta_{G}}(h g, \mu)=\left\{\left(\left(T_{g} L_{h} v, \rho\right),\left(T_{h g}^{*} L_{h^{-1}} \beta, \eta\right)\right) \in\left(T_{h g} G \times \mathfrak{g}^{*}\right) \times\left(T_{h g}^{*} G \times \mathfrak{g}\right) \mid\right. \\
\left(T_{g} L_{h} v, \rho\right) \in \Delta_{G \times \mathfrak{g}^{*}}(h g, \mu), \text { and }\left\langle T_{h g}^{*} L_{h^{-1}} \beta, T_{g} L_{h} w\right\rangle+\langle\sigma, \eta\rangle \\
\left.=\omega(h g, \mu)\left(\left(T_{g} L_{h} v, \rho\right),\left(T_{g} L_{h} w, \sigma\right)\right) \text { for all }\left(T_{g} L_{h} w, \sigma\right) \in \Delta_{G \times \mathfrak{g}^{*}}(h g, \mu)\right\} .
\end{aligned}
$$

\section{Reduction of induced distributions}

Recall the induced distribution $\Delta_{G \times \mathfrak{g}^{*}} \subset T\left(G \times \mathfrak{g}^{*}\right)$ from $\Delta_{G}$ can be locally given by

$$
\Delta_{G \times \mathfrak{g}^{*}}=\{(g, \mu, v, \rho) \mid g \in U, \quad v \in \Delta(g)\} .
$$

Since $\Delta_{G \times \mathfrak{g}^{*}}$ is left-invariant, it follows

$$
\Delta_{G \times \mathfrak{g}^{*}}(h g, \mu)=\Delta_{G \times \mathfrak{g}^{*}}(g, \mu)
$$

and taking quotients by $G$ at each $(e, \mu) \in G \times \mathfrak{g}^{*}$ yields

$$
\Delta_{G \times \mathfrak{g}^{*}}(e, \mu) / G=\mathfrak{g}^{\Delta} \oplus \mathfrak{g}^{*},
$$

where

$$
\mathfrak{g}^{\Delta}=\{\xi \in \mathfrak{g} \mid \xi \in \Delta(e)\}
$$

is a constraint subspace of $\mathfrak{g} \cong T_{e} G$.

\section{Dirac reduction}

Because of the $G$-invariance of $\mathcal{D}_{\Delta_{G}}$, an induced Dirac structure on $G \times \mathfrak{g}^{*}$

$$
\mathcal{D}_{\Delta_{G}} \subset T\left(G \times \mathfrak{g}^{*}\right) \oplus T^{*}\left(G \times \mathfrak{g}^{*}\right)
$$

may be identified with

$$
\mathcal{D}_{\Delta_{G}} \subset\left(G \times \mathfrak{g}^{*}\right) \times\left(V \oplus V^{*}\right),
$$

which is uniquely determined by its value at the identity as

$$
\begin{aligned}
\mathcal{D}_{\Delta_{G}}(e, \mu)= & \left\{((\xi, \rho),(\nu, \eta)) \in V \oplus V^{*} \mid(\xi, \rho) \in \mathfrak{g}^{\Delta} \oplus \mathfrak{g}^{*},\right. \\
& \text { and } \left.\langle\nu, \zeta\rangle+\langle\sigma, \eta\rangle=\omega(e, \mu)((\xi, \rho),(\zeta, \sigma)) \text { for all }(\zeta, \sigma) \in \mathfrak{g}^{\Delta} \oplus \mathfrak{g}^{*}\right\} .
\end{aligned}
$$


In view of the invariance of the induced Dirac structure, a structure, which we write $\mathcal{D}_{\Delta_{G}}^{/ G}(\mu)$, may be induced, for each $\mu \in \mathfrak{g}^{*}$, on the quotient space as

$$
\begin{aligned}
\mathcal{D}_{\Delta_{G}}^{/ G}(\mu)=\{((\xi, \rho),(\nu, \eta)) & \in V \oplus V^{*} \mid(\xi, \rho) \in \mathfrak{g}^{\Delta} \oplus \mathfrak{g}^{*}, \\
\text { and }\langle\nu, \zeta\rangle+\langle\sigma, \eta\rangle & \left.=\omega_{\mu}^{/ G}((\xi, \rho),(\zeta, \sigma)) \text { for all }(\zeta, \sigma) \in \mathfrak{g}^{\Delta} \oplus \mathfrak{g}^{*}\right\},
\end{aligned}
$$

where $\omega_{\mu}^{/ G}$ is the $\mu$-dependent symplectic structure on $V=\mathfrak{g} \oplus \mathfrak{g}^{*}$ given in Eq. (4.7).

Proposition 7.1. For fixed $\mu \in \mathfrak{g}^{*}, \mathcal{D}_{\Delta_{G}}^{/ G}(\mu)$ in Eq. (7.1) is given by

$$
\mathcal{D}_{\Delta_{G}}^{\prime G}(\mu)=\left\{((\xi, \rho),(v, \xi)) \in V \oplus V^{*} \mid \xi \in \mathfrak{g}^{\Delta}, \quad v+\rho-\operatorname{ad}_{\xi}^{*} \mu \in\left(\mathfrak{g}^{\Delta}\right)^{\circ}\right\} .
$$

Proof: Suppose that the condition (7.1) holds, namely, that

$$
\langle v, \zeta\rangle+\langle\sigma, \eta\rangle=\omega_{\mu}^{/ G}((\xi, \rho),(\zeta, \sigma))
$$

for all $(\zeta, \sigma) \in \mathfrak{g}^{\Delta} \oplus \mathfrak{g}^{*}$. First, using (4.7) and setting $\sigma=0$, gives

$$
\langle v+\rho, \zeta\rangle=\left\langle\mu, \operatorname{ad}_{\xi} \zeta\right\rangle \quad \text { for all } \zeta \in \mathfrak{g}^{\Delta} .
$$

So, one gets

$$
v+\rho-\operatorname{ad}_{\xi}^{*} \mu \in\left(\mathfrak{g}^{\Delta}\right)^{\circ} .
$$

Second, set $\zeta=0$ and then

$$
\langle\sigma, \eta-\xi\rangle=0 \quad \text { for all } \quad \sigma \in \mathfrak{g}^{*} .
$$

Hence, we have

$$
\eta=\xi \in \mathfrak{g}^{\Delta} .
$$

The converse is shown in the same way.

The induced Dirac structure on $V=\mathfrak{g} \oplus \mathfrak{g}^{*}$

As in Section 4, we have the following important theorem showing that the reduction of the induced Dirac structure on $T^{*} G$ gives a $\mu$-dependent Dirac structure on $V=\mathfrak{g} \oplus \mathfrak{g}^{*}$ induced from $\mathfrak{g}^{\Delta} \subset \mathfrak{g}$.

THEOREM 7.2. For fixed $\mu \in \mathfrak{g}^{*}$, the induced structure $\mathcal{D}_{\Delta_{G}}^{/ G}(\mu)$ given by (7.1), or equivalently, (7.2), is a Dirac structure on $V=\mathfrak{g} \oplus \mathfrak{g}^{*}$.

Proof: One can simply prove that for fixed $\mu$, Eq. (7.1) is a special case of the construction of a Dirac structure on a symplectic manifold $P$ given by Eq. (2.1), with $P=V$, with the symplectic structure $\omega_{\mu}^{/ G}$ and with $\mathfrak{g}^{\Delta} \oplus \mathfrak{g}^{*} \subset V$.

We shall give another direct proof of this theorem as follows. Noting that the symmetric paring on $V \oplus V^{*}$ is given by

$$
\langle\langle((w, \gamma),(\delta, u)), \quad((\xi, \rho),(\nu, \xi))\rangle=\langle v, w\rangle+\langle\gamma, \xi\rangle+\langle\delta, \xi\rangle+\langle\rho, u\rangle,
$$


the orthogonal space of $\mathcal{D}_{\Delta_{G}}^{/ G}(\mu)$ is given by, for each $\mu \in \mathfrak{g}^{*}$,

$$
\begin{aligned}
\left(\mathcal{D}_{\Delta_{G}}^{/ G}\right)^{\perp}(\mu) & =\left\{((w, \gamma),(\delta, u)) \in V \oplus V^{*} \mid\right. \\
& \left.\langle v, w\rangle+\langle\gamma, \xi\rangle+\langle\delta, \xi\rangle+\langle\rho, u\rangle=0, \quad \xi \in \mathfrak{g}^{\Delta}, \quad v+\rho-\operatorname{ad}_{\xi}^{*} \mu \in\left(\mathfrak{g}^{\Delta}\right)^{\circ}\right\} .
\end{aligned}
$$

What we want to prove is that $\mathcal{D}_{\Delta_{G}}^{/ G}(\mu)=\left(\mathcal{D}_{\Delta_{G}}^{/ G}\right)^{\perp}(\mu)$. Let us first check that $\mathcal{D}_{\Delta_{G}}^{/ G}(\mu) \subset\left(\mathcal{D}_{\Delta_{G}}^{/ G}\right)^{\perp}(\mu)$. Let $((w, \gamma),(\delta, w)) \in \mathcal{D}_{\Delta_{G}}^{/ G}(\mu)$, where $w \in \mathfrak{g}^{\Delta}$ and $\gamma+\delta-$ $\operatorname{ad}_{w}^{*} \mu \in\left(\mathfrak{g}^{\Delta}\right)^{\circ}$. Since $\xi \in \mathfrak{g}^{\Delta}$ and $v+\rho-\operatorname{ad}_{\xi}^{*} \mu \in\left(\mathfrak{g}^{\Delta}\right)^{\circ}$, we have

$$
\begin{aligned}
\langle v, w\rangle+\langle\gamma, \xi\rangle+\langle\delta, \xi\rangle+\langle\rho, w\rangle & =\langle v+\rho, w\rangle+\langle\gamma+\delta, \xi\rangle \\
& =\left\langle\alpha+\operatorname{ad}_{\xi}^{*} \mu, w\right\rangle+\left\langle\beta+\operatorname{ad}_{w}^{*} \mu, \xi\right\rangle \\
& =\left\langle\mu, \operatorname{ad}_{\xi} w\right\rangle+\left\langle\mu, \operatorname{ad}_{w} \xi\right\rangle \\
& =0,
\end{aligned}
$$

where $\alpha=v+\rho-\operatorname{ad}_{\xi}^{*} \mu$ and $\beta=\gamma+\delta-\operatorname{ad}_{w}^{*} \mu$. Therefore, $\mathcal{D}_{\Delta_{G}}^{\prime G}(\mu) \subset\left(\mathcal{D}_{\Delta_{G}}^{\prime G}\right)^{\perp}(\mu)$.

Conversely, let us check that $\left(\mathcal{D}_{\Delta_{G}}^{/ G}\right)^{\perp}(\mu) \subset \mathcal{D}_{\Delta_{G}}^{/ G}(\mu)$. Let $((w, \gamma),(\delta, u)) \in$ $\left(\mathcal{D}_{\Delta_{G}}^{/ G}\right)^{\perp}(\mu)$. Setting $\xi=0$ and $v+\rho=\operatorname{ad}_{\xi}^{*} \mu$, one has

$$
\begin{aligned}
\langle v, w\rangle+\langle\rho, u\rangle & =\left\langle\operatorname{ad}_{\xi}^{*} \mu-\rho, w\right\rangle+\langle\rho, u\rangle \\
& =\langle\rho, u-w\rangle \\
& =0
\end{aligned}
$$

for all $\rho$. So, one obtains $u=w$. Next, let $\xi$ be arbitrary and let $v+\rho=\operatorname{ad}_{\xi}^{*} \mu$. Noting $w=u$, one has

$$
\begin{aligned}
\langle v+\rho, w\rangle+\langle\gamma+\delta, \xi\rangle & =\left\langle\operatorname{ad}_{\xi}^{*} \mu, w\right\rangle+\langle\gamma+\delta, \xi\rangle \\
& =\left\langle\mu, \operatorname{ad}_{\xi} w\right\rangle+\langle\gamma+\delta, \xi\rangle \\
& =-\left\langle\mu, \operatorname{ad}_{w} \xi\right\rangle+\langle\gamma+\delta, \xi\rangle \\
& =\left\langle-\operatorname{ad}_{w}^{*} \mu+\gamma+\delta, \xi\right\rangle \\
& =0
\end{aligned}
$$

for all $\xi \in \mathfrak{g}^{\Delta}$. So, we have $\gamma+\delta-\operatorname{ad}_{w}^{*} \mu \in\left(\mathfrak{g}^{\Delta}\right)^{\circ}$. Hence $\left(\mathcal{D}_{\mu}^{/ G}\right)^{\perp} \subset \mathcal{D}_{\mu}^{/ G}$.

Finally, $\left(\mathcal{D}_{\mu}^{/ G}\right)^{\perp}=\mathcal{D}_{\mu}^{/ G}$ and thus, $\mathcal{D}_{\Delta_{G}}^{/ G}(\mu)$ is a $\mu$-dependent Dirac structure on $V=\mathfrak{g} \oplus \mathfrak{g}^{*}$ induced from $\mathfrak{g}^{\Delta} \subset \mathfrak{g}$.

\section{Euler-Poincaré-Suslov reduction}

In this section, making use of the Dirac reduction, we will develop the reduction of an implicit Lagrangian system $\left(L, \Delta_{G}, X\right)$, where a left invariant constraint distribution $\Delta_{G} \subset T G$ is given. In particular, we will show how a reduced implicit 
Lagrangian system can be obtained in an implicit form of the so-called EulerPoincaré-Suslov equations and we also illustrate an example of the Suslov problem of rigid body systems with nonholonomic constraints.

\section{Implicit Lagrangian systems on $T G \oplus T^{*} G$}

Let $L$ be a $G$-invariant Lagrangian (possibly degenerate) on $T G$. Let $\left(L, \Delta_{G}, X\right)$ be an implicit Lagrangian system, which satisfies

$$
(X, \mathfrak{D} L) \in D_{\Delta_{G}},
$$

where $X: T G \oplus T^{*} G \rightarrow T T^{*} G$ is a partial vector field on $T^{*} G, \mathfrak{D} L$ is the Dirac differential of $L$ and $D_{\Delta_{G}}$ is the induced Dirac structure.

\section{Coordinate representations}

The canonical two-form $\Omega$ is given in coordinates by

$$
\Omega\left(\left(g, p, u_{1}, \alpha_{1}\right),\left(g, p, u_{2}, \alpha_{2}\right)\right)=\left\langle\alpha_{2}, u_{1}\right\rangle-\left\langle\alpha_{1}, u_{2}\right\rangle,
$$

and the induced Dirac structure may be expressed, in coordinates, by

$$
D_{\Delta_{Q}}(g, p)=\left\{((g, p, \dot{g}, \dot{p}),(g, p, \alpha, w)) \mid \dot{g} \in \Delta(g), w=\dot{g} \text {, and } \alpha+\dot{p} \in \Delta^{\circ}(g)\right\} \text {. }
$$

Writing $X(q, v, p)=(g, p, \dot{g}, \dot{p})$ and $\mathfrak{D} L=(g, \partial L / \partial v,-\partial L / \partial g, v)$ together with $p=\partial L / \partial v$, and using the local expressions for the canonical symplectic form and the Dirac differential, the condition for an implicit Lagrangian system $(X, \mathfrak{D} L) \in D_{\Delta_{G}}$ reads that

$$
\left\langle-\frac{\partial L}{\partial g}, u\right\rangle+\langle\alpha, v\rangle=\langle\alpha, \dot{g}\rangle-\langle\dot{p}, u\rangle
$$

for all $u \in \Delta(g)$ and all $\alpha$, where $(u, \alpha)$ are the local representatives of a point in $T_{(g, p)} T^{*} G$. Since this holds for all $u \in \Delta(g)$ and all $\alpha$, it follows

$$
p=\frac{\partial L}{\partial v}, \quad \dot{g}=v \in \Delta(g), \quad \dot{p}-\frac{\partial L}{\partial g} \in \Delta^{\circ}(g),
$$

which are the local expressions for the implicit Lagrangian system on $T G \oplus T^{*} G$.

\section{Euler-Poincaré-Suslov reduction}

Let us give the definition of reduction of an implicit Lagrangian system associated with the induced Dirac structure on $T^{*} G$.

DEFINITION 8.1 (Euler-Poincaré-Suslov reduction). Let $\left(L, \Delta_{G}, X\right)$ be an implicit Lagrangian system, which is associated with the Dirac structure $D_{\Delta_{G}}$ induced from $\Delta_{G} \subset T G$. Let $\mathcal{D}_{\Delta_{G}}^{/ G}(\mu)$ be the $\mu$-dependent reduced Dirac structure on $V=\mathfrak{g} \oplus \mathfrak{g}^{*}$ associated with a constraint space $\mathfrak{g}^{\Delta} \subset \mathfrak{g}$, which is given in Eq. (7.1), or equivalently 
(7.2). The reduced partial vector field $\mathcal{X}^{/ G}: \mathfrak{g} \oplus \mathfrak{g}^{*} \rightarrow \mathfrak{g}^{*} \times\left(\mathfrak{g} \oplus \mathfrak{g}^{*}\right)$ is locally written as in Eq. (5.2). Let $l=L \mid \mathfrak{g}: \mathfrak{g} \rightarrow \mathbb{R}$ denote the reduced Lagrangian.

Then, the reduction of the implicit Lagrangian system $\left(L, \Delta_{G}, X\right)$ is given by a triple $\left(l, \mathfrak{g}^{\Delta}, \mathcal{X}^{/ G}\right)$ that satisfies

$$
\left(\mathcal{X}^{/ G}(\eta, \mu), \mathfrak{D}^{/ G} l(\eta)\right) \in \mathcal{D}_{\Delta_{G}}^{/ G}(\mu),
$$

where $\mathfrak{D}^{/ G} l$ is the reduced Dirac differential for $l$ given in Eq. (5.1) for all $\eta \in \mathfrak{g}$; again note that equality of base points requires that, in addition, $\mu=\mathbb{F} l(\eta)$.

Definition 8.2. A solution curve of $\left(l, \mathfrak{g}^{\Delta}, \mathcal{X}^{/ G}\right)$ is a curve $(\eta(t), \mu(t))$ in $\mathfrak{g}^{\Delta} \oplus \mathfrak{g}^{*}$ such that it is an integral curve of the partial vector field $\mathcal{X}^{/ G}$, where $\mu(t)=\mathbb{F} l(\eta(t))$.

PROPOSITION 8.3. An integral curve $(\eta(t), \mu(t))$ of the reduced implicit Lagrangian system $\left(l, \mathfrak{g}^{\Delta}, \mathcal{X}^{/ G}\right)$ satisfies the following equations:

$$
\mu=\frac{\delta l}{\delta \eta}, \quad \xi=\eta \in \mathfrak{g}^{\Delta}, \quad \dot{\mu}-\operatorname{ad}_{\xi}^{*} \mu \in\left(\mathfrak{g}^{\Delta}\right)^{\circ},
$$

where $\left(\mathfrak{g}^{\Delta}\right)^{\circ} \subset \mathfrak{g}^{*}$ is the annihilator of the constraint subspace $\mathfrak{g}^{\Delta}$.

Proof: Substituting $\mathcal{X}^{/ G}(\eta, \mu)=(\mu, \xi, \dot{\mu})$ and $\mathfrak{D}^{/ G} l(\eta)=(\delta l / \delta \eta, 0, \eta)$ into the condition of Eq. (8.2), for each $\eta \in \mathfrak{g}^{\Delta}$ and with fixed $\mu=\delta l / \delta \eta \in \mathfrak{g}^{*}$ in view of Eq. (7.2), it immediately reads

$$
((\xi, \dot{\mu}),(0, \eta)) \in \mathcal{D}_{\Delta_{G}}^{/ G}(\mu),
$$

and hence

$$
\xi=\eta \in \mathfrak{g}^{\Delta}, \quad \dot{\mu}-\operatorname{ad}_{\xi}^{*} \mu \in\left(\mathfrak{g}^{\Delta}\right)^{\circ},
$$

Thus, we obtain Eq. (8.3).

The set of equations of motion in Eq. (8.3) is the local expression for the reduction of the implicit Lagrangian system given in Eq. (8.1), which is an implicit analog of Euler-Poincaré-Suslov equations (see Bloch [7]). Then, we shall call Eq. (8.3) implicit Euler-Poincaré-Suslov equations on $\mathfrak{g}^{\Delta} \oplus \mathfrak{g}^{*}$.

\section{Energy conservation of implicit Lagrangian systems}

Let $\left(L, \Delta_{G}, X\right)$ be an implicit Lagrangian system, where $G$ is a Lie group, $L$ is a left invariant Lagrangian on $T G$ and $X: T G \oplus T^{*} G \rightarrow T T^{*} G$ is a partial vector field on $T^{*} G$. Let $\Delta_{G}$ be a left invariant distribution on $G$ and define the constraint momentum space $P \subset T^{*} G$ by $P=\mathbb{F} L\left(\Delta_{G}\right)$. Define an energy function $E$ on $T G \oplus T^{*} G$ by, for $(g, v, p) \in T G \oplus T^{*} G$,

$$
E(g, v, p)=\langle p, v\rangle-L(g, v) .
$$

Let $(g(t), v(t), p(t))$ in $\Delta_{G} \oplus P \subset T G \oplus T^{*} G$ be a solution curve of $\left(L, \Delta_{G}, X\right)$, where $p(t)=(\partial L / \partial v)(t)$. 
Recall from Yoshimura and Marsden [50] that for the implicit Lagrangian system $\left(L, \Delta_{G}, X\right)$, energy conservation holds along the solution curve $(g(t), v(t), p(t))$; that is, the energy $E(q(t), v(t), p(t))$ is constant in time. This is shown as follows:

$$
\begin{aligned}
\frac{d}{d t} E & =\langle\dot{p}, v\rangle+\langle p, \dot{v}\rangle-\frac{\partial L}{\partial g} \dot{g}-\frac{\partial L}{\partial v} \dot{v} \\
& =\left\langle\dot{p}-\frac{\partial L}{\partial g}, v\right\rangle,
\end{aligned}
$$

which vanishes since $\dot{g}=v \in \Delta(g)$ and since $\dot{p}-\partial L / \partial g \in \Delta^{\circ}(q)$.

\section{Energy conservation of reduced implicit Lagrangian systems}

Recall that the group $G$ acts on curves $(g(t), v(t), p(t))$ in $T G \oplus T^{*} G$, by simultaneously left translating on each factor by the left-action and the tangent and cotangent lifts. Explicitly, the action of an element $h \in G$ is given on a curve $g(t) \in G, v(t) \in T_{g(t)} G$ and $p(t) \in T_{g(t)}^{*} G$, by

$$
h \cdot(g(t), v(t), p(t))=\left(h g(t), T_{g(t)} L_{h} \cdot v(t), T_{h g(t)}^{*} L_{h^{-1}} \cdot p(t)\right),
$$

where $T_{g(t)} L_{h}: T_{g(t)} G \rightarrow T_{h g(t)} G$ is the tangent of the left translation map $L_{h}$ : $G \rightarrow G ; g(t) \mapsto h g(t)$ at the point $g(t)$ and $T_{h g(t)}^{*} L_{h^{-1}}: T_{g(t)}^{*} G \rightarrow T_{h g(t)}^{*} G$ is the dual of the map $T_{h g(t)} L_{h^{-1}}: T_{h g(t)} G \rightarrow T_{g(t)} G$. The energy $E$ is invariant under the action of $G$ since $L$ is $G$-invariant and one easily checks that the momentum function $\langle p(t), v(t)\rangle$ is also $G$-invariant. Hence one can define the reduced energy $e$ on $\mathfrak{g} \oplus \mathfrak{g}^{*}$ by, for $(\eta, \mu) \in \mathfrak{g} \oplus \mathfrak{g}^{*}$,

$$
e(\eta, \mu)=\langle\mu, \eta\rangle-l(\eta)
$$

where $l=L \mid \mathfrak{g}$ is the reduced Lagrangian on $\mathfrak{g}$ and $\langle\mu, \eta\rangle$ is the reduced momentum function on $\mathfrak{g} \oplus \mathfrak{g}^{*}$. Let $\mathfrak{g}^{\Delta} \subset \mathfrak{g}$ be the reduction of $\Delta_{G} \subset T G$ and let $\mathcal{X} / G$ : $\mathfrak{g} \oplus \mathfrak{g}^{*} \rightarrow \mathfrak{g}^{*} \times\left(\mathfrak{g} \oplus \mathfrak{g}^{*}\right)$ be the reduction of the partial vector field $X$.

We have the following proposition of energy conservation for reduced implicit Lagrangian systems.

Proposition 8.4. Let $\left(l, \mathfrak{g}^{\Delta}, \mathcal{X}^{/ G}\right)$ be the reduced implicit Lagrangian system, which is the reduction of a given implicit Lagrangian system $\left(L, \Delta_{G}, X\right)$. Let $(\eta(t), \mu(t))$ be a solution curve in $\mathfrak{g}^{\Delta} \oplus \mathfrak{g}^{*} \subset \mathfrak{g} \oplus \mathfrak{g}^{*}$ of $\left(l, \mathfrak{g}^{\Delta}, \mathcal{X}^{/ G}\right)$, where $\mu(t)=(\delta l / \delta \eta)(t)$. Then conservation of energy holds along the solution curve $(\eta(t), \mu(t))$; that is, the (reduced) energy $e(\eta(t), \mu(t))$ is constant in time.

Proof: By the definition of the reduced energy $e$, it follows, by noting $\mu(t)=$ 
$(\delta l / \delta \eta)(t)$

$$
\begin{aligned}
\frac{d}{d t} e(\eta, \mu) & =\langle\dot{\mu}, \eta\rangle+\langle\mu, \dot{\eta}\rangle-\frac{\delta l}{\delta \eta} \dot{\eta} \\
& =\left\langle\mu-\frac{\delta l}{\delta \eta}, \dot{\eta}\right\rangle+\left\langle\operatorname{ad}_{\xi}^{*} \mu, \eta\right\rangle \\
& =\left\langle\operatorname{ad}_{\xi}^{*} \mu, \xi\right\rangle,
\end{aligned}
$$

which vanishes since $\eta=\xi \in \mathfrak{g}^{\Delta}$ and since $\dot{\mu}-\operatorname{ad}_{\xi}^{*} \mu \in\left(\mathfrak{g}^{\Delta}\right)^{\circ}$. Thus, the (reduced) energy $e$ is constant in time.

\section{Examples of implicit Euler-Poincaré-Suslov equations}

Let us illustrate the Euler-Poincaré-Suslov reduction theory with an example of the Suslov problem for the case $G=\mathrm{SO}(3)$. As to the Suslov problem, refer to, for instance, [34, 53, 7, 10].

Now, we consider the Suslov problem, which is an Euler top with a nonholonomic constraint, which represents a rigid body rotating with a fixed point such that at each moment of time the projection of the angular velocity in some direction fixed in the body is equal to zero.

Let $L: T \mathrm{SO}(3) \rightarrow \mathbb{R}$ be a left invariant Lagrangian (the kinetic energy), i.e., $L(g, v)=l\left(T_{g} L_{g-1} v\right)$, where $l: \mathfrak{s o}(3) \rightarrow \mathbb{R}$ is the reduced Lagrangian, which is locally given by

$$
l(\Sigma)=\frac{1}{2}\langle\Sigma, \mathbb{I} \Sigma\rangle,
$$

where $\Sigma=T_{g} L_{g^{-1}} v \in \mathfrak{s o}(3)$ and $\mathbb{I}$ denotes the inertia tensor of a rigid body. The Suslov problem is a class of nonholonomic systems with a constraint distribution

$$
\mathfrak{s o}(3)^{\Delta}=\{\Sigma \in \mathfrak{s o}(3) \mid\langle\mathbf{A}, \Sigma\rangle=0\},
$$

where $\Sigma$ is the body angular velocity and $\mathbf{A}$ is a fixed element of the dual Lie algebra $\mathfrak{s o}(3)^{*}$. Here, $\langle\cdot, \cdot\rangle$ stands for the natural paring between the Lie algebra and its dual. Since the subspace $\mathfrak{s o}(3)^{\Delta}$ is not necessarily a subalgebra, the constraint is nonholonomic. Then, the Suslov problem may be represented by the reduced implicit Lagrangian system $\left(l, \mathfrak{s o}(3)^{\Delta}, \mathcal{X}^{/ G}\right)$ as in equation (8.2). Namely, the system equations for the Suslov problem can be expressed by the implicit Euler-Poincaré-Suslov equations, which are given in this example by

$$
\Pi_{i}=\frac{\partial l}{\partial \Sigma^{i}}, \quad \Omega^{i}=\Sigma^{i}, \quad \dot{\Pi}_{i}-C_{j i}^{k} \Omega^{j} \Pi_{k}=\lambda A_{i}, \quad i, j, k=1,2,3 .
$$

In the above, $C_{j i}^{k}$ are the structure constants of $\mathfrak{s o}(3)$ and $\lambda$ is the Lagrange multiplier. Further, $\Sigma=\Sigma^{i} \mathbf{e}_{i}, \Omega=\Omega^{i} \mathbf{e}_{i} \in \mathfrak{s o}(3)^{\Delta}, \mathbf{A}=A_{i} \mathbf{e}^{i} \in \mathfrak{s o}(3)^{*}$, and $\Pi=\Pi_{i} \mathbf{e}^{i} \in \mathfrak{s o}(3)^{*}$ denotes the body angular momentum, where $\mathbf{e}_{i}, i=1,2,3$ form a basis for $\mathfrak{s o}(3)$ and $\mathbf{e}^{i}$ form a basis for $\mathfrak{s o}(3)^{*}$. 
As demonstrated in [53], let us choose $\mathbf{e}_{3}=\mathbf{A} /|\mathbf{A}|$ as the third vector of the body frame and let us pick up two independent vectors $\mathbf{e}_{1}, \mathbf{e}_{2}$ that are orthogonal to $\mathbf{e}_{3}$ in the kinetic energy metric. Then, one has the constraint

$$
\Omega_{3}=0 .
$$

Note that $\mathbf{e}_{1}, \mathbf{e}_{2}$ and $\mathbf{e}_{3}$ are not necessarily orthogonal relative to the standard metric in $\mathfrak{s o}(3) \cong \mathbb{R}^{3}$ unless $\mathbf{e}_{3}$ spans an eigenspace of the inertia tensor $\mathbb{I}=I_{i j} \mathbf{e}_{i} \otimes \mathbf{e}_{j}$. This implies that the structure constants $C_{j i}^{j}$ are not equal to zero. Noting that the components of the inertia tensor $I_{13}$ and $I_{23}$ are zero, hence, it follows

$$
\Pi_{1}=I_{11} \Sigma^{1}+I_{12} \Sigma^{2}, \quad \Pi_{2}=I_{21} \Sigma^{1}+I_{22} \Sigma^{2}, \quad \Pi_{3}=I_{33} \Sigma^{3} .
$$

The implicit Euler-Poincaré-Suslov equations are given in matrix form by

$$
\left(\begin{array}{c}
\dot{\Pi}_{1} \\
\dot{\Pi}_{2} \\
\dot{\Pi}_{3}
\end{array}\right)=\left(\begin{array}{c}
C_{21}^{1} \Omega^{2} \Pi_{1}+C_{21}^{2} \Omega^{2} \Pi_{2} \\
C_{12}^{1} \Omega^{1} \Pi_{1}+C_{12}^{2} \Omega^{1} \Pi_{2} \\
C_{13}^{1} \Omega^{1} \Pi_{1}+C_{13}^{2} \Omega^{1} \Pi_{2}+C_{23}^{1} \Omega^{2} \Pi_{1}+C_{23}^{2} \Omega^{2} \Pi_{2}
\end{array}\right)+\left(\begin{array}{c}
0 \\
0 \\
\lambda|\mathbf{A}|
\end{array}\right)
$$

and with

$$
\Omega_{1}=\Sigma_{1}, \quad \Omega_{2}=\Sigma_{2}, \quad \Omega_{3}=\Sigma_{3}=0 .
$$

Notice that $\Pi_{3}=0$ since $\Omega_{3}=\Sigma_{3}=0$. By eliminating the Lagrange multiplier, we finally obtain

$$
\left(\begin{array}{c}
\dot{\Pi}_{1} \\
\dot{\Pi}_{2}
\end{array}\right)=\left(\begin{array}{c}
C_{21}^{1} \Omega^{2} \Pi_{1}+C_{21}^{2} \Omega^{2} \Pi_{2} \\
C_{12}^{1} \Omega^{1} \Pi_{1}+C_{12}^{2} \Omega^{1} \Pi_{2}
\end{array}\right)
$$

and with

$$
\Omega_{1}=\Sigma_{1}, \quad \Omega_{2}=\Sigma_{2}, \quad \Pi_{1}=I_{11} \Sigma^{1}+I_{12} \Sigma^{2}, \quad \Pi_{2}=I_{21} \Sigma^{1}+I_{22} \Sigma^{2} .
$$

The above implicit Euler-Poincaré-Suslov equations are equivalent with the equations in [53].

For the case in which we choose $\mathbf{e}_{3}$ as an eigenvector of the inertia tensor such that $\mathbb{I} \mathbf{e}_{3}=I_{33} \mathbf{e}_{3}$ and in which we also choose $\mathbf{e}_{1}$ and $\mathbf{e}_{2}$ as the two remaining eigenvectors, the basis $\mathbf{e}_{1}, \mathbf{e}_{2}, \mathbf{e}_{3}$ in $\mathfrak{s o}(3)$ is orthogonal with respect to both the standard and the kinetic energy metrics, where $C_{12}^{3}=C_{23}^{1}=C_{31}^{2}=-C_{21}^{3}=-C_{32}^{1}=-C_{13}^{2}=1$ and $C_{j i}^{k}=0$ otherwise. Then, we get

$$
\dot{\Pi}_{1}=0, \quad \dot{\Pi}_{2}=0 .
$$

Thus, all the solutions of the reduced system are relative equilibria.

\section{Lie-Poisson-Suslov reduction}

For the case in which a given Lagrangian is regular, or when a Hamiltonian is given, we can develop a Hamiltonian analogue of Euler-Poincaré-Suslov reduction. This section, gives a reduction procedure for an implicit Hamiltonian system 
$(X, \mathbf{d} H) \in D_{\Delta_{G}}$ associated with an induced Dirac structure on $D_{\Delta_{G}}$, which we shall call Lie-Poisson-Suslov reduction. It is also shown that this reduction procedure may be also useful in the analysis of the Suslov problem in nonholonomic mechanics.

\section{The implicit Hamiltonian system on a Lie group}

Let $H$ be a $G$-invariant Hamiltonian on $T^{*} G$. Let $\left(H, \Delta_{G}, X\right)$ be an implicit Hamiltonian system, which satisfies

$$
(X, \mathbf{d} H) \in D_{\Delta_{G}},
$$

where $X$ is a vector field on $T^{*} G, \mathbf{d} H$ is the differential of $H$ and $D_{\Delta_{G}}$ is the induced Dirac structure.

\section{Coordinate representations}

Recall that the canonical two-form $\Omega$ is given, in coordinates, by

$$
\Omega\left(\left(g, p, u_{1}, \alpha_{1}\right),\left(g, p, u_{2}, \alpha_{2}\right)\right)=\left\langle\alpha_{2}, u_{1}\right\rangle-\left\langle\alpha_{1}, u_{2}\right\rangle,
$$

and the induced Dirac structure may be expressed, in coordinates, by

$$
D_{\Delta_{G}}(g, p)=\left\{((g, p, \dot{g}, \dot{p}),(g, p, \alpha, w)) \mid \dot{g} \in \Delta(g), w=\dot{g}, \text { and } \alpha+\dot{p} \in \Delta^{\circ}(g)\right\} \text {. }
$$

Writing $X=(g, p, \dot{g}, \dot{p})$ and $\mathbf{d} H=(g, p, \partial H / \partial g, \partial H / \partial p)$, and using the local expressions for the canonical symplectic form and the differential of $H$, the condition for an implicit Hamiltonian system $(X, \mathbf{d} H) \in D_{\Delta_{G}}$ reads

$$
\left\langle\frac{\partial H}{\partial g}, u\right\rangle+\left\langle\alpha, \frac{\partial H}{\partial p}\right\rangle=\langle\alpha, \dot{g}\rangle-\langle\dot{p}, u\rangle
$$

for all $u \in \Delta(g)$ and all $\alpha$, where $(u, \alpha)$ are the local representatives of a point in $T_{(g, p)} T^{*} G$. Since this holds for all $u \in \Delta(g)$ and all $\alpha$, we obtain the local expressions for the implicit Hamiltonian system as

$$
\dot{g}=\frac{\partial H}{\partial p} \in \Delta(g), \quad \dot{p}+\frac{\partial H}{\partial g} \in \Delta^{\circ}(g) .
$$

\section{Lie-Poisson-Suslov reduction}

Let us develop the reduced implicit Hamiltonian systems associated with the reduction of an induced Dirac structure on $\mathfrak{g} \oplus \mathfrak{g}^{*}$, which we shall call Lie-PoissonSuslov reduction.

Let $\left(H, \Delta_{G}, X\right)$ be an implicit Hamiltonian system, which satisfies $(X, \mathbf{d} H) \in$ $D_{\Delta_{G}}$, where $H$ is a left invariant Hamiltonian on $T^{*} G, \Delta_{G} \subset T G$ is a given distribution and $X$ is a vector field on $T^{*} G$.

Definition 9.1 (Lie-Poisson-Suslov reduction). Let $\left(H, \Delta_{G}, X\right)$ be an implicit Hamiltonian system, which is associated with the Dirac structure $D_{\Delta_{G}}$ on $T^{*} G$ 
induced from $\Delta_{G} \subset T G$. Let $h: \mathfrak{g}^{*} \rightarrow \mathbb{R}$ be the reduced Hamiltonian defined by $h=H \mid \mathfrak{g}^{*}$ and let $\mathfrak{g}^{\Delta} \subset \mathfrak{g}$ be a constraint space. Denote by $\mathcal{X}^{/ G}$ the partial vector field defined in Eq. (6.3), and $\mathcal{D}_{\Delta_{G}}^{/ G}(\mu)$ denote the reduction of the induced Dirac structure on $V=\mathfrak{g} \oplus \mathfrak{g}^{*}$, which is defined at each $\mu \in \mathfrak{g}^{*}$, by Eq. (7.1), or equivalently Eq. (7.2).

Then, the reduction of the implicit Hamiltonian system $\left(H, \Delta_{G}, X\right)$ is given by a triple $\left(h, \mathfrak{g}^{\Delta}, \mathcal{X}^{/ G}\right)$ that satisfies, at each $\mu \in \mathfrak{g}^{*}$,

$$
\left(\mathcal{X}^{/ G}(\mu), \mathbf{d}^{/ G} h(\mu)\right) \in \mathcal{D}_{\Delta_{G}}^{/ G}(\mu),
$$

where $\mathbf{d}^{/ G} h$ is the reduction of the differential of $H$, which is locally given in Eq. (6.1).

Definition 9.2. A solution curve of $\left(h, \mathfrak{g}^{\Delta}, \mathcal{X}^{/ G}\right)$ is a curve $\mu(t)$ in $\mathfrak{g}^{*}$, which is an integral curve of the partial vector field $\mathcal{X}^{/ G}=(\mu, \xi(\mu), \dot{\mu})$ such that $\xi(t)=\mathbb{F} h(\mu(t)) \in \mathfrak{g}^{\Delta}$.

Proposition 9.3. The reduced implicit Hamiltonian systems $\left(h, \mathfrak{g}^{\Delta}, \mathcal{X}^{/ G}\right)$ induces the local expressions

$$
\xi=\frac{\delta h}{\delta \mu} \in \mathfrak{g}^{\Delta}, \quad \dot{\mu}-\operatorname{ad}_{\xi}^{*} \mu \in\left(\mathfrak{g}^{\Delta}\right)^{\circ},
$$

where $\left(\mathfrak{g}^{\Delta}\right)^{\circ} \subset \mathfrak{g}^{*}$ is the annihilator of the constraint subspace $\mathfrak{g}^{\Delta}$.

Proof: Substituting Eqs. (6.1) and Eq. (6.3) into the condition of Eq. (9.1), namely,

$$
\left(\mathcal{X}^{/ G}(\mu), \mathbf{d}^{/ G} h(\mu)\right) \in \mathcal{D}_{\Delta_{G}}^{/ G}(\mu),
$$

it immediately reads, in view of Eq. (7.2), Eq. (9.2).

We shall call the set of equations in Eq. (9.2) implicit Lie-Poisson-Suslov equations on $\mathfrak{g}^{\Delta} \oplus \mathfrak{g}^{*}$.

\section{Examples of implicit Lie-Poisson-Suslov equations}

For the case in which a given Lagrangian is regular, we can construct the reduced implicit Hamiltonian system associated with the reduced implicit Lagrangian system in the context of the Lie-Poisson-Suslov reduction. Here, let us demonstrate the Lie-Poisson-Suslov reduction together with the same example of the Suslov problem of nonholonomic rigid body systems for the case $G=\mathrm{SO}(3)$ as we showed in the Euler-Poincaré-Suslov reduction.

Let $H: T^{*} \mathrm{SO}(3) \rightarrow \mathbb{R}$ be a left invariant Hamiltonian, that is, $H(g, p)=$ $h\left(T_{e}^{*} L_{g} p\right)$, where $h: \mathfrak{s o}(3)^{*} \rightarrow \mathbb{R}$ is the reduced Hamiltonian locally given by

$$
h(\Pi)=\frac{1}{2}\left\langle\Pi, \mathbb{I}^{-1} \Pi\right\rangle,
$$


where $\Pi=T_{e}^{*} L_{g} p \in \mathfrak{s o}(3)^{*}$.

Associated with the nonholonomic constraint distribution $\mathfrak{s o}(3)^{\Delta} \subset \mathfrak{s o}(3)$ given in Eq. (8.4), the rigid body system can be represented in the context of the reduced implicit Hamiltonian system $\left(h, \mathfrak{s o}(3)^{\Delta}, \mathcal{X}^{/ G}\right)$. Then, the reduced implicit Hamiltonian system for the Suslov problem may be expressed by the implicit Lie-Poisson-Suslov equations given in Eq. (9.2), which are locally given, in this example, by

$$
\Omega^{i}=\frac{\partial h}{\partial \Pi_{i}}, \quad \dot{\Pi}_{i}-C_{j i}^{k} \Omega^{j} \Pi_{k}=\lambda A_{i}, \quad i, j, k=1,2,3,
$$

where $\Omega=\Omega^{i} \mathbf{e}_{i} \in \mathfrak{s o}(3)^{\Delta}$.

Let us choose $\mathbf{e}_{3}=\mathbf{A} /|\mathbf{A}|$ as the third vector of the body frame. Then, we have the constraint $\Omega_{3}=0$, and hence the implicit Lie-Poisson-Suslov equations are finally given in matrix by

$$
\left(\begin{array}{c}
\dot{\Pi}_{1} \\
\dot{\Pi}_{2}
\end{array}\right)=\left(\begin{array}{c}
C_{21}^{1} \Omega^{2} \Pi_{1}+C_{21}^{2} \Omega^{2} \Pi_{2} \\
C_{12}^{1} \Omega^{1} \Pi_{1}+C_{12}^{2} \Omega^{1} \Pi_{2}
\end{array}\right)
$$

and with

$$
\Omega^{1}=I^{11} \Pi_{1}+I^{21} \Pi_{2}, \quad \Omega^{2}=I^{12} \Pi_{1}+I^{22} \Pi_{2},
$$

which are equivalent with the equations of motion given in [53].

\section{Conclusions}

In this paper, we have presented a reduction theory for induced $G$-invariant Dirac structures on the cotangent bundle $T^{*} G$ of a Lie group $G$ along with its associated reduced implicit Lagrangian and Hamiltonian systems. First, we have shown how an implicit analogue of the Euler-Poincaré equations, namely, the implicit Euler-Poincaré equations, can be developed as the reduction of the implicit EulerLagrange equations in the context of reduction of the Hamilton-Pontryagin variational principle. Second, we have developed a Lie-Dirac reduction theory for reducing the canonical Dirac structure on $T^{*} G$; namely, we have constructed a $\mu$-dependent reduced Dirac structure $\mathcal{D}_{\mu}^{/ G}$ on $\mathfrak{g} \oplus \mathfrak{g}^{*}$, which includes both the coadjoint orbit symplectic structure and the Lie-Poisson structure. Then, we have incorporated $\mathcal{D}_{\mu}^{\prime G}$ into the reduction of standard implicit Lagrangian systems to develop Euler-PoincaréDirac reduction, which eventually induces the implicit Euler-Poincaré equations. We have also investigated the Hamiltonian analogue of Euler-Poincaré-Dirac reduction, namely, Lie-Poisson-Dirac reduction for the case of a given regular Lagrangian, or Hamiltonian, which is the reduction of the standard implicit Hamiltonian system, consistent with the Lie-Poisson variational principle. Further, we have established reduction of an induced Dirac structure on $T^{*} G$ for the general case $\Delta_{G} \subset T^{*} G$, and we have demonstrated how it can be incorporated into the Suslov problem in nonholonomic mechanics. We have constructed a $\mu$-dependent reduced Dirac structure 
$\mathcal{D}_{\Delta_{G}}^{/ G}(\mu)$ on $\mathfrak{g} \oplus \mathfrak{g}^{*}$, which has been incorporated into the context of Euler-PoincaréSuslov reduction for reducing an implicit Lagrangian system as well as into the context of Lie-Poisson-Suslov reduction for reducing an implicit Hamiltonian system. Lastly, we have demonstrated that the implicit Euler-Poincaré-Suslov equations as well as the implicit Lie-Poisson-Suslov equations can be naturally obtained from this reduction theory.

Some interesting topics for future work that may be relevant with this paper are as follows:

- Reduction of an induced Dirac structure on the cotangent bundle of a manifold $Q$ for the case of principal bundle with a Lie group $G$ acting freely and properly on $Q$; especially, how it is relevant with an implicit analogue of Lagrangian reduction and Lagrange-Poincaré equations (see, for instance, [18]).

- The relationship with Lie algebroids and associated Lagrangian reduction that were noted in Weinstein [49]; how implicit Euler-Poincaré equations can be related with Lie algebroids; the link with Lie bialgebroids that are relevant with Drinfel'd [26].

- The Courant algebroid in relation with Dirac bundle reduction and its associated reduced implicit Lagrangian and Hamiltonian systems with a nontrivial (nonintegrable) distribution on $Q$.

- The formal variational analysis for integrable systems (see [28]) in relation with, for instance, Hamiltonian structures in infinite LC transmission lines.

- Applications to rigid body problems, ideal fluid dynamics and so on; for instance, how implicit Euler-Poincaré equations can be applied to degenerate cases, such as the Maxwell-Vlasov equations, as in [17].

- Development of Dirac reductions in discrete mechanics and applications to numerical integration and Hamilton-Pontryagin variational integrators, both unreduced and reduced in particular (see, for instance, [30] and Bou-Rabee and Marsden [11]).

\section{Acknowledgement}

We are very grateful to Tudor Ratiu, Alan Weinstein and Troy Smith for providing useful remarks and suggestions. We also thank the reviewers for their helpful suggestions and comments.

\section{REFERENCES}

[1] R. Abraham and J. E. Marsden: Foundations of Mechanics. Benjamin-Cummings Publ. Co, 1978, Updated 1985 version, reprinted by Persius Publishing, second edition.

[2] R. Abraham, J. E. Marsden, and T. S. Ratiu: Manifolds, Tensor Analysis and Applications, volume 75 of Applied Mathematical Sciences, second edition, Springer, New York, 1988.

[3] L. Bates and J. Sniatycki: Nonholonomic reduction, Rep. Math. Phys. 32 (1993), 99-115.

[4] G. Blankenstein: Implicit Hamiltonian Systems: Symmetry and Interconnection, Ph.D. Dissertation. University of Twente, 2000.

[5] G. Blankenstein and A. J. van der Schaft: Symmetry and reduction in implicit generalized Hamiltonian systems. Rep. Math. Phys. 47 (1) (2001), 57-100. 
[6] G. Blankenstein and T. S. Ratiu: Singular reduction of implicit Hamiltonian systems, Rep. Math. Phys. 53 (2004), 211-260.

[7] A. M. Bloch: Nonholonomic Mechanics and Control, volume 24 of Interdisciplinary Applied Mathematics. Springer, New York, 2003, with the collaboration of J. Baillieul, P. Crouch and J. Marsden, and with scientific input from P. S. Krishnaprasad, R. M. Murray and D. Zenkov.

[8] A. M. Bloch and P. E. Crouch: Representations of Dirac structures on vector spaces and nonlinear L-C circuits. In Differential Geometry and Control (Boulder, CO, 1997), Proc. Sympos. Pure Math. 64 (1997), 103-117, Amer. Math. Soc., Providence, RI, 1997.

[9] A. M. Bloch, P. S. Krishnaprasad, J. E. Marsden, and R. Murray: Nonholonomic mechanical systems with symmetry, Arch. Rational Mech. Anal. 136 (1996), 21-99.

[10] A. M., J. Bloch E. Marsden and D. V. Zenkov: Nonholonomic dynamics, Notices of the AMS. 52 (2006), 302-329.

[11] N. Bou-Rabee and J. E. Marsden: Reduced Hamilton-Pontryagin variational integrators, submitted for publication, 2007.

[12] H. Bursztyn, M. Cavalcanti, R. Gil and M. Gualtieri: Reduction of Courant algebroids and generalized complex structures, math. DG/0509640, 2006.

[13] H. Bursztyn, M. Cavalcanti, R. Gil and M. Gualtieri: Generalized Kähler and hyper-Kähler quotients, math. DG/0702104, 2007.

[14] H. Bursztyn and M. Crainic: Dirac structures, momentum maps, and quasi-Poisson manifolds. In J. E. Marsden and T. S. Ratiu, editors. The Breath of Symplectic and Poisson Geometry, pages 1-40. Birkhäuser, Boston, 2006.

[15] H. Bursztyn, M. Crainic, A. Weinstein and C. Zhu: Integration of twisted Dirac brackets, Duke Math. Journal. 123 (3) (2004), 549-604.

[16] H. Bursztyn and O. Radko: Gauged equivalence of Dirac structures and symplectic groupoids, Ann. Inst. Fourier (Grenoble) 53 (1) (2003), 309-337.

[17] H. Cendra, D. D. Holm, M. J. W. Hoyle and J. E. Marsden: The Maxwell-Vlasov equations in Euler-Poincaré form, J. Math. Phys. 39 (1998), 3138-3157.

[18] H. Cendra, J. E. Marsden, S. Pekarsky, and T. S. Ratiu: Variational principles for Lie-Poisson and Hamilton-Poincaré equations, Moscow Mathematical Journal 3 (2003), 833-867.

[19] H. Cendra, J. E. Marsden and T. S. Ratiu: Lagrangian reduction by stages, volume 152 of Memoirs, American Mathematical Society, Providence, R.I., 2001.

[20] H. Cendra, J. E. Marsden and T. S. Ratiu: Geometric mechanics, Lagrangian reduction and nonholonomic systems. In Enquist, B. and W. Schmid, editors, Mathematics Unlimited-2001 and Beyond, p. 221-273. Springer, New York, 2001.

[21] H. Cendra, J. E. Marsden and T. S. Ratiu: Cocycles, compatibility, and Poisson brackets for complex fluids. In Capriz, G. and P. Mariano (editors), Advances in Multifield Theories with Substructures, Modeling and Simulation in Science, Engineering and Technology Series, p. 51-73. Birkhäuser, Boston, 2003.

[22] D. Chang E. and J. E. Marsden: Reduction of controlled Lagrangian and Hamiltonian systems with symmetry, SIAM. J. Control and Optimization 43 (2003), 277-300.

[23] T. J. Courant: Dirac manifolds, Trans. Amer. Math. Soc. 319 (1990), 631-661.

[24] T. J. Courant: Tangent Dirac structures, J. Phys. A: Math. Gen. 23 (1990), 5153-5168.

[25] T. Courant and A. Weinstein, Beyond Poisson structures. In Action hamiltoniennes de groupes. Troisième théorème de Lie (Lyon, 1986), volume 27 of Travaux en Cours, p. 39-49, Hermann, Paris, 1988.

[26] V. G. Drinfel'd: Hamiltonian structures on Lie groups, Lie bialgebras and the geometric meaning of the classical Yang-Baxter equations, Soviet Math. Dokl. 27 (1) (1983), 68-71.

[27] I. Dorfman: Dirac structures of integrable evolution equations, Physics Letters A. 125 (5) (1987), $240-246$.

[28] I. Dorfman: Dirac Structures and Integrability of Nonlinear Evolution Equations. Number 94j:58081, in Nonlinear Science: Theory and Applications, Wiley, Chichester, 1993.

[29] $\mathrm{Yu}$ N. Fedorov and V. V. Kozlov: Various aspects of $n$-dimensional rigid body dynamics. In Amer. Math. Soc. Transl. Series 2. 168 (1995), 141-171. 
[30] L. Kharevych, Weiwei, Y. Tong, E. Kanso, J. E. Marsden, P. Schröder and M. Desbrun: Geometric, variational integrators for computer animation, Eurographics/ACM SIGGRAPH Symposium on Computer Animation, 1-9, 2006.

[31] J. Koiller: Reduction of some classical nonholonomic systems with symmetry, Arch. Rat. Mech. Anal. 118 (1992), 113-148.

[32] W. S. Koon and J. E. Marsden: The Hamiltonian and Lagrangian approaches to the dynamics of nonholonomic systems, Rep. Math. Phys. 40 (1997), 21-62.

[33] W. S. Koon and J. E. Marsden: Poisson reduction for nonholonomic mechanical systems with symmetry, Rep. Math. Phys. 42 (1998), 101-134.

[34] V. V. Kozlov: Invariant measures of the Euler-Poincaré equations on Lie algebras, Funct. Anal. Appl. 22 (1988), 58-59.

[35] G. H. Livens: On Hamilton's principle and the modified function in analytical dynamics, Proc. Roy. Soc. Edingburgh 39 (1919), 113.

[36] Z.-J., A. Liu Weinstein and P. Xu: Dirac structures and Poisson homogeneous spaces, Comm. Math. Phys. 192 (1998), 121-144.

[37] J. E. Marsden: Lectures on Mechanics, volume 174 of London Mathematical Society Lecture Note Series, Cambridge University Press, Cambridge, 1992.

[38] J. E. Marsden, G. Misiolek, J.-P. Ortega, M. Perlmutter and T.S. Ratiu: Hamiltonian Reduction by Stages, volume 1913 of Lecture Notes in Mathematics, Springer, 2007.

[39] J. E. Marsden and T. S. Ratiu: Reduction of Poisson manifold, Lett. Math. Phys. 11 (1986), $161-169$.

[40] J. E. Marsden and T. S. Ratiu: Introduction to Mechanics and Symmetry, volume 17 of Texts in Applied Mathematics, second edition, Springer, 1999.

[41] J. E. Marsden, Ratiu, T. S. and J. Scheurle: Reduction theory and the Lagrange-Routh equations, J. Math. Phys. 41 (6) (2000), 3379-3429.

[42] J. E. Marsden and A. Weinstein: Reduction of symplectic manifolds with symmetry, Rep. on Math.Phys. 5 (1974), 121-130.

[43] J. E. Marsden and A. Weinstein: Coadjoint orbits, vortices and Clebsch variables for incompressible fluids, Physica D. 7 (1983), 305-323.

[44] J. Marsden E. and A. Weinstein: Comments on the history, theory, and applications of symplectic reduction, Quantization of Singular Symplectic Quotients, Birkhäuser (N. Landsman, M. Pflaum and M. Schlichenmaier, eds.), 1-20, Boston, 2001.

[45] L. S, V. Pontryagin G. Boltyanskiǔ, R. V. Gamkrelidze and E. F. Mishchenko: Mathematical Theory of Optimal Processes, translated by Trirogoff, K. N. Wiley-Interscience, New York, 1962.

[46] H. J. Sussmann: An introduction to the coordinate-free maximum principle, In Geometry of Feedback and Optimal Control (B. Jackubczyk and W. Respondek, eds.), Monographs Textbooks Pure. Appl. Math. 207, 463-557, M. Dekker (1998).

[47] A. J. van der Schaft and B. M. Maschke: The Hamiltonian formulation of energy conserving physical systems with external ports, Archiv für Elektronik und Übertragungstechnik 49 (1995), 362-371.

[48] A. J. van der Schaft: Implicit Hamiltonian systems with symmetry, Rep. Math. Phys. 41 (1998), $203-221$.

[49] A. Weinstein: Lagrangian mechanics and groupoids, Field Institute Communications 7 (1996), $207-231$.

[50] H. Yoshimura and J. E. Marsden: Dirac structures in Lagrangian mechanics Part I: Implicit Lagrangian systems, J. Geom. and Phys. 57 (2006), 133-156.

[51] H. Yoshimura and J. E. Marsden, Dirac structures in Lagrangian mechanics Part II: Variational structures, J. Geom. and Phys. 57 (2006), 209-250.

[52] H. Yoshimura and J. E. Marsden: Dirac structures and the Legendre transformation for implicit Lagrangian and Hamiltonian systems, Proc. IFAC Conference on Lagrangian and Hamiltonian Methods in Nonlinear Control, 2006 (to appear).

[53] D. V. Zenkov and A. M. Bloch: Dynamics of the $n$-dimensional Suslov problem, J. Geom. and Phys. 34 (2006), 121-136. 